\title{
Advanced Role of Neutrophils in Common Respiratory Diseases
}

\author{
Jinping Liu, ${ }^{1}$ Zhiqiang Pang, ${ }^{2}$ Guoqiang Wang, ${ }^{2}$ Xuewa Guan, ${ }^{2}$ Keyong Fang, ${ }^{3}$ \\ Ziyan Wang, ${ }^{2}$ and Fang Wang ${ }^{2}$ \\ ${ }^{1}$ Institute of Frontier Medical Science, Jilin University, Changchun 130021, China \\ ${ }^{2}$ Department of Pathogen Biology, College of Basic Medical Sciences, Jilin University, Changchun 130021, China \\ ${ }^{3}$ Department of Pharmacology, College of Basic Medical Sciences, Jilin University, Changchun 130021, China
}

Correspondence should be addressed to Fang Wang; wf@jlu.edu.cn

Received 1 February 2017; Revised 22 March 2017; Accepted 16 April 2017; Published 15 May 2017

Academic Editor: Michael Schnoor

Copyright (c) 2017 Jinping Liu et al. This is an open access article distributed under the Creative Commons Attribution License, which permits unrestricted use, distribution, and reproduction in any medium, provided the original work is properly cited.

Respiratory diseases, always being a threat towards the health of people all over the world, are most tightly associated with immune system. Neutrophils serve as an important component of immune defense barrier linking innate and adaptive immunity. They participate in the clearance of exogenous pathogens and endogenous cell debris and play an essential role in the pathogenesis of many respiratory diseases. However, the pathological mechanism of neutrophils remains complex and obscure. The traditional roles of neutrophils in severe asthma, chronic obstructive pulmonary diseases (COPD), pneumonia, lung cancer, pulmonary fibrosis, bronchitis, and bronchiolitis had already been reviewed. With the development of scientific research, the involvement of neutrophils in respiratory diseases is being brought to light with emerging data on neutrophil subsets, trafficking, and cell death mechanism (e.g., NETosis, apoptosis) in diseases. We reviewed all these recent studies here to provide you with the latest advances about the role of neutrophils in respiratory diseases.

\section{Introduction}

With the changing of global environment, especially the increased air pollution worldwide, respiratory diseases are becoming a main killer to human health. According to recent researches, asthma is ranked as the 14th most important chronic disease, affecting 334 million individuals of all ages worldwide [1]. Lung is the leading cancer site in males, comprising $17 \%$ of the total new cancer cases and $23 \%$ of the total cancer deaths [2]. As for COPD, affecting 64 million people all over the world, it would be the third most common cause of death by 2030 [3, 4]. Communityacquired pneumonia is a common cause of sepsis, leading to 10 million deaths annually [5]. While epidemiology data of idiopathic pulmonary fibrosis (IPF) worldwide cannot be obtained, IPF incidence is still increasing and carries a high risk of respiratory failure and death [6]. Respiratory diseases not only increase the economic burden of global health care but also cause a terrible effect on the quality of daily life. Although the precise treatment of respiratory diseases has made a great progress [7-9], the pathogenesis of them still needs further elucidation.

Innate together with adaptive immunity, as natural systematic defensive barrier, is composed of immune organs, cells, and cytokines $[10,11]$. The innate immunity is a natural defense that shapes in the process of long-time biological evolution $[12,13]$. As the first barrier to defend infection, innate immunity participates in the resistance to pathogenic invasion and the clearance of aging, injured and even mutant cells nonspecifically. Innate immunity was firstly reported in the development of immunology and was becoming the focus of immunological research in recent twenty years, especially after the discovery of various kinds of pattern recognition receptors (PRRs) and innate lymphoid cells (ILCs) [14-17]. When the exogenous threats cannot be removed by innate immunity successfully, adaptive immunity will take part in the important defensive battle. Adaptive immunity system including humoral and cellular immunity often plays a leading role in the final clearance of invasive pathogens. The executors are T lymphoid cells and B lymphoid cells, 
who can both recognize antigens specifically. Different immune cells can exert protective and defensive effect synergistically with the help of multiple cytokines and protein molecules. The mechanism of adaptive immunity has been being gradually clarified since the birth of immunology. Various monoclonal antibody medicines related with adaptive immunity such as rituximab and infliximab have brought a wonderful curative effect in many refractory diseases including respiratory diseases $[18,19]$.

Traditionally, neutrophils, originating in bone marrow stem cells, had only been considered as a kind of innate immune cell [20]. As an essential component of innate immunity, neutrophils play an important role in killing pathogens and removing cellular debris [21]. The migration and activation of neutrophils could cause inflammation and sensitization directly or indirectly. Inflammation caused by self-immune system is really important for the solution of infection and clearance of pathogens. But the persistent inflammation in respiratory system frequently leads to some adverse diseases such as asthma, COPD, and pulmonary fibrosis. In addition, neutrophils can synergize with lymphocytes and other granulocytes, such as Th2/Th17 and eosinophils, to participate in not only innate but also adaptive immune process and promote airway inflammation $[22,23]$. The interaction between neutrophils and other immune cells, endogenous composition, and foreign matter is very complex and being clarified thoroughly.

There have been more and more studies on the role of neutrophils in respiratory diseases. Recently, exosomes, neutrophil extracellular traps (NETs), deriving from neutrophil and the higher autophagy of neutrophils have been reported in multiple respiratory diseases $[24,25]$. Despite that the pathogeneses of respiratory diseases are being studied extensively, there is still a long way to go to clarify the complexity and heterogeneity, especially the participation of various immune components in the development of respiratory diseases. In this review, the latest progress of neutrophils in respiratory disease such as asthma, COPD, pneumonia, lung cancer, pulmonary fibrosis, bronchitis, and bronchiolitis will be summarized.

\section{Asthmatic Neutrophils}

2.1. What Is Neutrophilic Asthma? As Global Initial for Asthma (GINA, updated in 2017) elucidated [26], asthma is a heterogeneous disease, always characterized by expiratory airflow limitation and chronic inflammation. Asthma is usually categorized as different phenotypes and endotypes according to its different clinical characteristics and distinct pathological mechanism. Traditionally, asthma was classified as four different phenotypes [27], eosinophilic, neutrophilic, mixed granulocytic asthma, and paucicellular asthma according to the cellular counts of sputum, bronchoalveolar lavage fluid (BALF), or peripheral blood [28]. For example, Jodie et al. distinguished asthmatics with neutrophil proportion in sputum over $61 \%$ as neutrophilic asthma [29]. However, more and more researches have demonstrated the instability of asthma phenotypes [30-32]. Neutrophil as an essential granulocyte has been reported by many investigators to play a critical role in many immunity-associated diseases including asthma, especially steroid-refractory severe asthma [33, 34]. High blood neutrophils counts are associated with an increased risk of moderate, but not severe asthma exacerbation [35]. At the same time, the neutrophil-predominant asthmatics also tend to show a lower bronchial lability [36].

\subsection{How Neutrophils Participate in Asthma?}

2.2.1. Chemotactic Activity of Neutrophils. Lower respiratory tracts used to be considered as sterile. But more and more evidence had already showed us the conflicted results [37]. Moraxella catarrhalis or a member of the Haemophilus or Streptococcus genera was discovered colonizing in the lower airways of asthmatics [38]. These species' colonization was associated with more differential sputum neutrophil counts and worse clinical disease status. The altered colonization would participate in the development of asthma phenotype. Infection of $H$. influenza could synergize with allergic airway diseases to induce Th17 immune responses that drive the development of neutrophilic asthma. The process above is mediated by IL-17 responses [39]. In addition, subclinical infection likely contributes to neutrophilic inflammation in airways [40].

Microbial components, which contain LPS and $\beta$-glucan, could synergistically cause neutrophilic asthma mediated by TLR-4 and dectin-1 [41], whose deficiency could significantly attenuate the recruitment of neutrophils induced by house dust mite (HDM) into airways [42]. Blood neutrophils from allergic asthma also show the chemotactic and phagocytic activities towards LPS and asthmatic serum [43]. Asthmatics challenged with inhaled Dermatophagoides pteronyssinus (DP) would promote the production of neutrophil chemotaxis [44]. Siew et al. demonstrated that neutrophil chemotaxis induced by smoke and other environmental stimulations could also be helpful for developing inflammation in airways [45].

As described above, smoking and other infectious factors can cause the accumulation of neutrophils in BALF. This is associated with the activation of phosphatidylinositol 3-kinase (PI3K) signal [46, 47]. Phosphatidylinositol 3-kinases (PI3Ks), as the key elements in the signaling cascades, play an important role in the chemotaxis of neutrophils [48]. In particular, PI3K $\delta$ and $\mathrm{PI} 3 \mathrm{~K} \gamma$ isoforms contribute to inflammatory cell recruitment and subsequent activation [49]. The traditional role of different PI3K isoforms in the chemotaxis of neutrophils had already been reviewed previously $[50,51]$. PI3K $\gamma$ deficiency could significantly reduce the influx of neutrophils into BALF [52]. PI3K $\delta$ inhibition may also prevent recruitment of neutrophils [53]. The PI3K-related inflammation and steroid insensitivity should partly be attributed to microRNA-21/PI3K/histone deacetylase 2 (HDAC2) axis as Kim et al. reported [54]. In addition, the activation of PI3K is accompanied with the release of all kinds of chemokines and cytokines, such as IL- 6 and IL-8, which are related with the increased chemotactic activity of neutrophils towards the inflamed sites $[55,56]$. Not only 


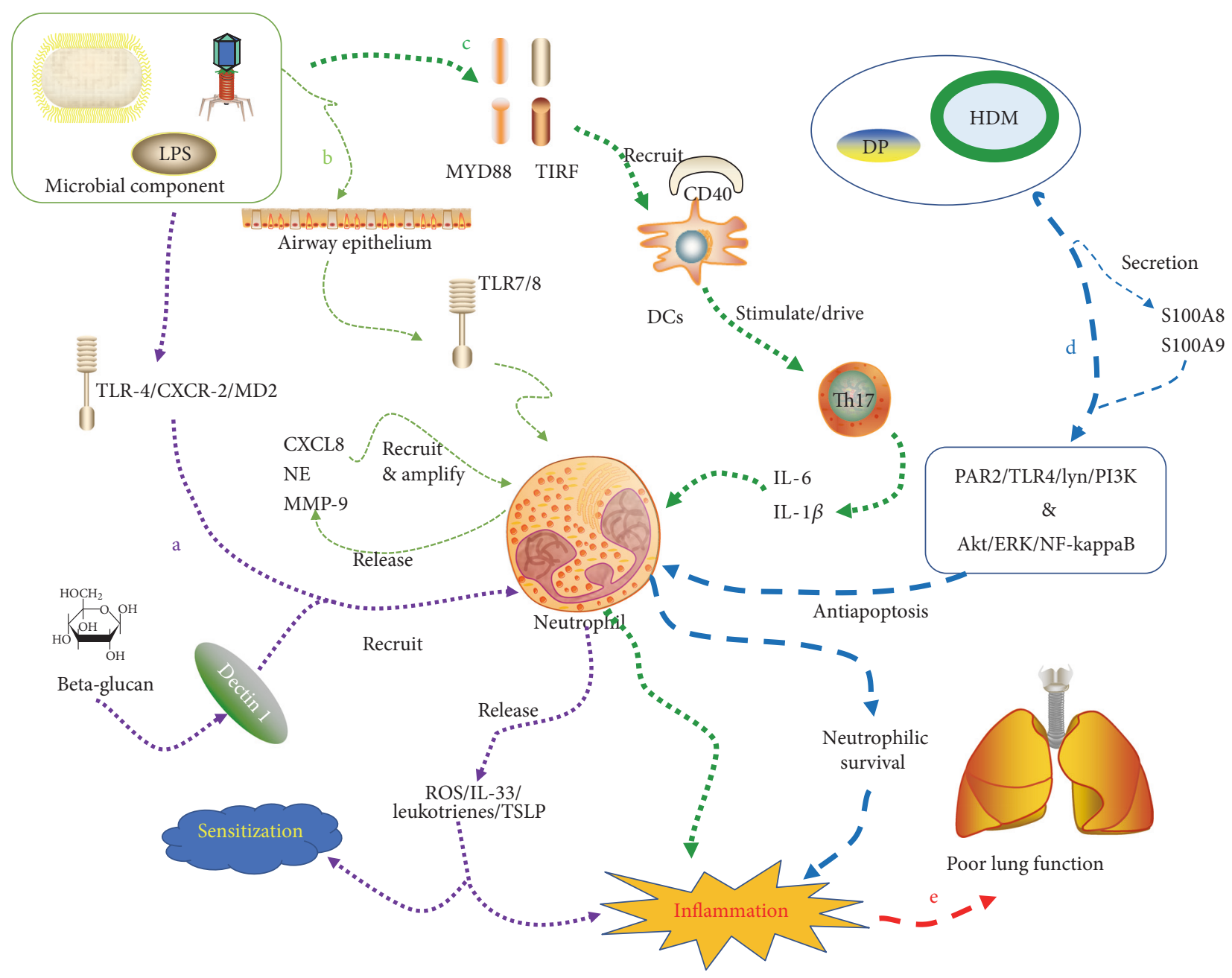

FIGURE 1: a: microbial component and environmental allergens such as LPS and $\beta$-glucan could recruit neutrophils via TLR-4/CXCR-2/MD2 and dectin-1. The recruited neutrophils could produce ROS/leukotrienes/IL-33/TSLP and cause inflammation or sensitization. b: the foreign allergens can act on airway epithelium and induce neutrophils to release CXCL8/NE/MMP-9, which is mediated by TLR-7/8. The released substance could amplify the recruitment of neutrophils in a positive feedback manner. c: microbial products and inhaled allergens can induce Th17 activated through TIRF and MYD88 by activating IRF-3, inducing I interferons, upregulating CD40 on DC, and finally releasing IL- 6 and IL- $1 \beta$ to act towards airway neutrophils. TIRF also contribute to Th17 responses to inhaled allergens by increasing recruitment of DCs and to drive Th17 cell differentiation. d: HDM-DP could induce the secretion of S100A8/S100A9. The combination of S100A8/S100A9 might activate TLR4/Lyn/PI3K/Akt/ERK/NF-kappaB pathway to produce an antiapoptotic effect on neutrophils. e: the stimulated or survival neutrophils can cause the persistence of inflammation. As a result, the lung function is getting poorer and poorer.

the chemotactic activity of neutrophils but also the concrete details of neutrophil activation mechanism have been making progress in recent years.

\subsubsection{Immune Interaction Related with Neutrophil} Activation. As previously reviewed [57], TIR-domaincontaining adapter-inducing interferon-beta (TRIF) played a key role in the induction of inflammatory mediators which could contribute to antiviral innate immune. Hsia et al. discovered that TRIF, as an essential component together with MYD88, could mediate microbial products (inhaled allergen) to induce Th17 activated. The process may include an activation of IRF-3, induction of I interferons, upregulation of CD40 on DCs or CD40L on T cells, and finally, the release of IL- 6 and IL- $1 \beta$, which are required for airway neutrophils [58]. The environmental stimulations could promote the production of IL-17. The expression level of IL-17 is also correlated with the expression of IL-8 and neutrophil numbers [45]. It was revealed that TRIF-CD40-Th17 axis participated in the IL-17-associated neutrophilic asthma. In addition, TRIF might also contribute to Th17 responses to inhaled allergens by increasing the recruitment of DCs to lung with potential to drive Th17 cell differentiation [58] (Figure 1, c). Similar to that, Siew et al. discovered that CSE (cigarette smoke extract), IL-17, and aeroallergens could act on human tracheal epithelial cells and further increase IL-6 and IL-8 production [45]. CD11b + DCs could sense some molecules in HDM extract and play a key role in the 
induction of HDM-induced allergic airway inflammation by inducing the expression of chemokine or chemokine receptors in DCs by expressing dectin-1 [42].

Rhinovirus infection could induce bronchial mucosal neutrophilia in subjects with asthma. Airway neutrophils during infection are positively related to virus load [59]. Recently, it has been demonstrated that LPS and viral infection could also promote the release of CXCL8/NE/MMP-9 from neutrophils, which is mediated by TLR7/8. CXCL8, as a potent chemoattractant for neutrophils released by a variety of cells including neutrophils, could amplify the recruitment in a positive feedback manner [60] (Figure 1, b). Several studies have also reported that CXCL8 levels in the airways inversely correlate with FEV1 in asthmatics [38, 61]. However, N-formyl-methionyl-leucyl-phenylalanine (fMLF), a bacterial-derived protein and ligand for fMLF receptor [62] could stimulate neutrophils from asthmatics to produce CXCL8, whose production is positively correlated with $\mathrm{FEV}_{1}$ and $\mathrm{FEV}_{1} / \mathrm{FVC}$. It showed that circulating neutrophils might be related to airflow limitation. These exteriorly conflicting reports tell us that neutrophils may be not the main source of CXCL8 in BALF [63]. In addition, Page et al. reported that challenging with German cockroach feces towards airway could migrate neutrophils into the airways and activate neutrophil cytokine production via TLR2 [64].

2.2.3. Inflammation Caused by Neutrophils. The inferior management of asthma in clinic practice is attributed to the persistent uncontrolled inflammation. Hosoki et al. report that allergens, such as pollen and cat dander, recruit neutrophils in a TLR-4/CXCR-2/MD2-dependent manner to the airways. The recruited neutrophils could produce ROS/leukotrienes/IL-33/TSLP (thymic stromal lymphopoietin) (Figure 1, a). All of these could cause the airway inflammation and allergic sensitization [65]. TLR4 expressed on hematopoietic cells is critical for neutrophilic airway inflammation following LPS exposure and for Th17-driven neutrophilic responses to the HDM lysates and ovalbumin (OVA). But at airway epithelial cells, TLR4 could also participate in the eosinophilic airway inflammation [66]. As described above, challenging with inhaled DP in asthmatics would not only increase the chemotaxis of neutrophils but also promote the production of ROS and the phagocytic activity, reflecting an enhanced systemic inflammation [44]. Moreover, apoptotic neutrophils in airway tissues could undergo secondary necrosis as the cause of inflammation [67].

TLR4 and cytokines seem to participate in the inflammatory process. The function of neutrophils from asthma patients was impaired with the lower levels of IL-8, IL- $1 \beta$, and TNF- $\alpha$ and decreased Tlr4 gene expression [68]. All of these changes would lead to increased susceptibility and severity of infections. But this was conflicted with the discovery reported by Baines et al. which claimed that noneosinophilic asthma had an elevated level of IL-8 [69]. Simpson et al. reported that clarithromycin, which had additionally been used to reduce neutrophilic inflammation in asthma, can significantly reduce airway concentrations of IL-8 and neutrophil accumulation and activation in the airways of patients with refractory asthma [70]. Corresponding to the reports above, the withdrawal of inhaled corticosteroid (ICS) can lead to an increase of neutrophils and IL- 8 in sputum [71]. The distinct role of IL-8 reported by researchers can be attributed to the different clinical research group design. In detail, noneosinophilic asthma is not equal to the neutrophilic asthma completely as previously described.

Recent investigation reported that a series of secretory protein (CCSP) could significantly reduce oxidative burst activity and increase phagocytosis of neutrophils [72]. CCSP could also enter neutrophils and alter their function. Secretoglobin protein, as a sort of CCSPs, has anti-inflammatory properties. Cote et al. reported that secretoglobin 1A1A could increase neutrophil oxidative burst and phagocytosis. Neutrophilic extracellular traps (NETs) are significantly reduced by secretoglobins $1 \mathrm{~A} 1$ and $1 \mathrm{~A} 1 \mathrm{~A}$. Their functional difference may contribute to the pathogenesis of recurrent asthma obstruction [73]. These proteins have the potential to be novel therapeutic targets in the future.

2.2.4. Prosurvival of Neutrophils. Granulocytes, including eosinophils and neutrophils, have the significant capacity to evoke tissue inflammation and remodeling. The removal of these granulocytes would contribute to the resolution of airway inflammation in asthma. Tian et al. discovered that promoted apoptosis of inflammatory cells, such as eosinophils and neutrophils would be essential for the clearance of allergen-induced airway inflammation, especially for corticosteroid-insensitive neutrophilic airway inflammation [74]. However, noneosinophilic asthma shows an enhancement of blood neutrophil chemotaxis and survival [69]. Uddin et al. enumerated the apoptotic neutrophils in sputum from asthmatic patients with different disease severities and found amongst a subset of neutrophilic asthmatics (>65\% PMNs) their sputum neutrophils inversely correlated with lung function (FEV1, \% predicted) due to unidentified factors present in sputum [75]. HDM-DP, as an allergen from our environment, could induce the secretion of myeloid-related protein 8 (MRP8, S100A8) and MRP14 (S100A9). The combination of them might activate TLR4/Lyn/PI3K/Akt/ERK/NF-kappaB pathway so as to produce an antiapoptotic effect on neutrophils [76] (Figure 1, d). The similar discovery was also reported by Lee et al. [77]. The survival of neutrophils in BALF would bring up a poor lung function, consistent with the decline of FEV1, as Sikkeland et al. discovered [78] (Figure 1, e).

2.3. Where Is the Potential Targeted Therapy? It is believed that the failure of targeting neutrophils could be attributed to an incomplete understanding of underlying mechanism of neutrophilic asthma [79]. New insights into emerging neutrophil biology and underlying mechanisms of neutrophil phenotype might come to be the evidences of precisionbased medicine. ICS is still the first-line medicine for ameliorating syndromes. Coinhalation of roflumilast and fluticasone, which reduces the counts of both neutrophils and eosinophils in BALF, could significantly improve the inflammatory condition in OVA-induced mice compared with the combination of formoterol and fluticasone [80]. 
Manually synthetic chemical drugs have an important curative effect on various diseases all along. Simvastatin, as an effective serum cholesterol-lowering agent could reduce the percentage of neutrophil in BALF and improve airway inflammation and remodeling in obese asthma mice [81]. Tamoxifen had a direct action on equine peripheral blood neutrophils and dampened the respiratory burst production [82]. Rosiglitazone (RSG), a peroxisome proliferatoractivated receptor- $\gamma$ agonist, has been reported to attenuate airway inflammation by inhibiting the proliferation of effector $\mathrm{T}$ cells in a murine model of neutrophilic asthma in vivo [48]. It can also downregulate the ratio of Treg and Th17 cells, inhibit the secretion of Th2 cytokines, and further inhibit the airway inflammatory response in asthma mice effectively [83]. AZD5069 as an antagonist of CXCR2, a receptor promoting neutrophils back to the inflamed airways, could reversibly reduce circulating neutrophils' count [84]. SCH527132, a selective CXCR2 receptor antagonist, can reduce sputum neutrophils and tend to improve the Asthma Control Questionaire scores of asthma [67].

Medicine from various plants has composed a large part in health care filed. Ligustrazine [85], water extract of $\mathrm{Hel}$ minthostachys zeylanica (L.) Hook [86], astragalin as an anti-inflammatory flavonoid present in persimmon leaves and green tea seeds [87], hydroethanolic extract $(70 \%)$ of M. longiflora (HEMI) [88], bufalin [89], and cordycepin [90] could target to the neutrophils, intervene the different inflammatory signaling pathways, and improve the prognosis of asthma. Biopharmaceutical has become a new treatment for asthma in recent years. Recombinant human activated protein C (rhAPC) could attenuate HDM + LPS-induced neutrophil migration in allergic asthma [91]. Another recombination protein, recombinant human IL-4, could inhibit airway inflammation in bronchial asthma by reducing the cytokines and inflammatory cells including neutrophils [92]. Medicine from plants and targeted biopharmaceuticals has a huge potential to play a major role in future medical field.

\section{Neutrophils in COPD}

3.1. Neutrophils Participate in COPD. The role of neutrophils in COPD is different from that in asthma [93]. In asthma, neutrophils are important only in some relatively rare and severe subtypes. However, neutrophils seem to always play a major role in COPD. The activation of neutrophils in the lung is directly correlated with the severity of symptoms [93]. The elevated neutrophil-lymphocyte ratio (NLR) can be used as a marker in the determination of increased inflammation and early detection of potential acute exacerbations in COPD patients. [94]. Although neutrophils in COPD patients' airway mucosa play a key role in antimicrobial defense theoretically as described in present review, a high number of neutrophils in patients' lungs are believed to correlate with poor prognosis [95]. It is well known to us all that neutrophil is an important part in maintaining our host defense and responding to injury and microbial infection. The removal of neutrophils can promote a return to homeostasis after its short-lived journey from circulation to injured or infected site. As Bratton et al. described, if not being removed, the dying neutrophils may even contribute to the ongoing inflammation, tissue damage, or autoimmune diseases by disintegrating and releasing various phlogistic cargoes [96].

3.2. The Role of Pathogen Infection and Proteases from Neutrophil in COPD. The pathogens' colonization, such as microbiota in lower respiratory airway, could interact with immune system. Airway bacterial loading at baseline correlates with sputum percent neutrophil count [97]. In COPD, exposure to bacterial pathogens could cause characteristic innate immune responses in peripheral blood monocytes and polymerphonuclear neutrophils (PMN), accompanied with the elevated protein expression of IL-8/IL-6/TNF- $\alpha$ / IFN- $\gamma$ [98] (Figure 2, b). At the same time, Guiot et al. also observed that COPD patients had higher IL-6, IL-8, TNF- $\alpha$, and MMP-9 in their induced sputum [99]. Treatment with levofloxacin in stable COPD could reduce the bacterial loading in a short time. This reduction is associated with the decrease of neutrophilic airway inflammation in patients with high airway bacterial loads [97].

Neutrophils contain a great deal of proteases, inflammatory mediators, and oxidants [100]. IL-22/IL-22R signaling pathway plays a pivotal role in antimicrobial defense. Influenza virus can promote the expression of IL-22R in human bronchial epithelia cells (Figure 2, a). Neutrophil-derived proteases may contribute to COPD by impairing the antimicrobial IL-22/IL-22R signaling pathway and decreasing the expression of antimicrobial effectors such as $\beta$-defensin- 2 . This process probably enhances pathogen replication and ultimately causes COPD exacerbations [95].

Neutrophil elastase (NE), one of the main proteases produced by neutrophils, plays an important role in inflammatory process. NE is able to increase the release of chemokines from epithelial cells with the activation of p38- $\alpha$-MAP-kinase. The production of these chemokines can be blocked by roflumilast $\mathrm{N}$-oxide combined with prostaglandin $E_{2}$ [101]. Neutrophil elastase-generated fragments of elastin (EL-NE) is different between stable and exacerbation of COPD patients. The serum level of EL-NE is associated with lung function [102]. Elastin breakdown mediated by neutrophil elastase is associated with COPD-induced inflammation [103]. $\alpha$-1-Antitrypsin (A1AT), as an endogenous inhibitor of NE, can limit lung damage. Its effect had been already reviewed by Meijer et al. [93]. Deficiency of it is known as a risk factor for lung function [93]. Geraghty et al. reported that bioactive A1AT could modulate phosphatase $2 \mathrm{~A}$, which played a key role in COPD and expressed on the neutrophils to prevent inflammatory and proteolytic responses triggered by TNF- $\alpha$ simulation in the lung [104]. Whether A1AT or its unknown analogues can be used as a potential novel medicine needs further scientific research and clinical validation.

3.3. Adverse Effect of Cigarette Smoke via Neutrophils. Cigarette smoking (CS), a main environmental trigger of COPD [93], can decrease sputum neutrophils significantly. Experiments in vitro showed that CS could induce necrotic 


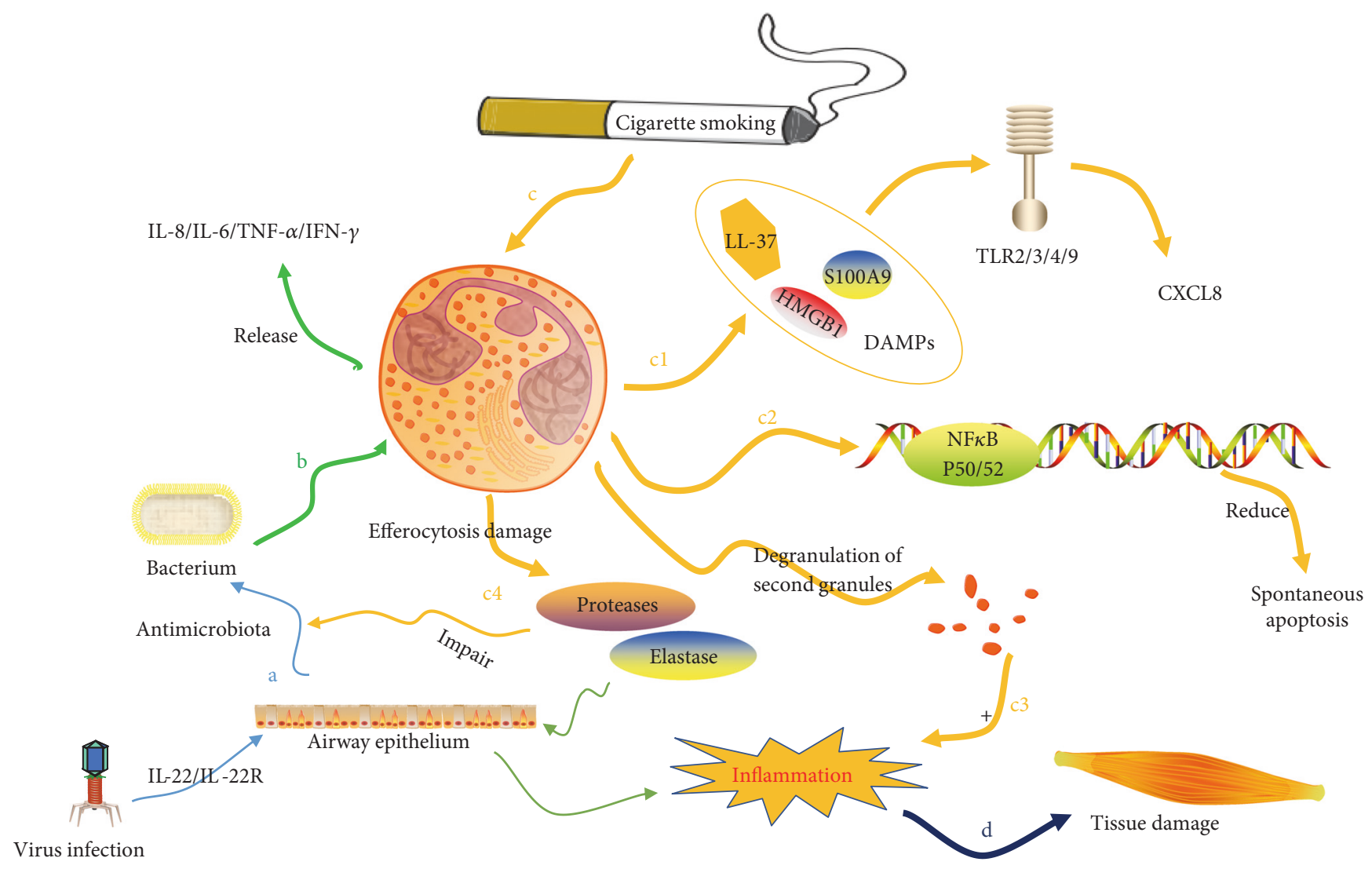

FIGURE 2: a: infection of virus can stimulate airway epithelium and produce antimicrobiota defense through IL-22/IL-22R signaling pathway. b: the colonization of bacterial pathogens could cause the release of IL-8/IL-6/TNF- $\alpha /$ IFN- $\gamma$. c1: cigarette smoking (CS) could induce the release of damage-associated molecular patterns (DAMPs). DAMPs can activate the innate immune system by binding to pattern recognition receptors (PRRs), such as TLR2, TLR4, and TLR9, and further induce CXCL8 release via TLR9 activation. c2: CS increase the expression of both p50 and p65 subunits of NF-kappaB in neutrophils and reduce the spontaneous apoptosis of neutrophils. c3: CS could cause the degranulation of secondary granules. This contributes to the accumulation of neutrophils and inflammation within the airways of smokers. c4: the efferocytosis damage caused by CS can increase the release of proteases and elastases. It not only impairs the antimicrobial defense but also (d) promotes the pulmonary inflammation and tissue degradation.

neutrophil cell death through mitochondrial dysfunction, apoptosis inhibition, and damage-associated molecular pattern (DAMP) release $[105,106]$. DAMPs can activate the innate immune system by binding to pattern recognition receptors (PRRs), such as TLR2, TLR4, and TLR9. DAMP signaling plays an important role in the activation of neutrophils during COPD exacerbations. Serum level of DAMP gene expression is increased during COPD exacerbations [106]. TLR2, TLR4, and NLRP3 expressions in neutrophils are increased during acute exacerbations of COPD compared with stable disease. The activation of TLR2/TLR4 can induce the activation and the migration of neutrophils and may thus contribute to the elevated airway inflammation during COPD exacerbations [107]. The detailed positive feedback loop between DAMPs and TLR4 and the function of neutrophils' TLR4/TLR3 in respiratory diseases had already been reviewed by other researchers [106-108]. The novel therapeutic strategies aimed at DAMPs and its receptors need more attention.

Normal human bronchial epithelial cells could release more CXCL-8 significantly when stimulated with the supernatants from CS-treated neutrophils [105]. While in
COPD patients, it was showed that there was an increase of inflammatory response and a decrease of PMNs apoptosis, which is independent from antiapoptotic cytokines such as CXCL-8 [109]. Mortaz et al. reported that cigarette smoking extract (CSE) could induce CXCL8 release via TLR9 activation [110] (Figure 2, c1). In addition, CSE could cause the degranulation of secondary granules. This may contribute to the accumulation of neutrophils and inflammation within the airways of smokers (Figure 2, c3). Furthermore, it promotes the pulmonary inflammation and tissue degradation [111].

Many kinds of membrane moleculars expressed on the surface of neutrophils are able to take part in mediating the biological activity resulted from cigarette smoke. Hoonhorst et al. reported that young individuals susceptible to COPD showed a significantly higher increase in the expression of Fc- $\gamma$-RII (CD32) in its active forms (A17 and A27) on neutrophils after smoking. This may indicate that systemic inflammation could participate in the early induction phase of COPD [112]. CD9, a transmembrane protein of the tetraspanin family, facilitates some pathogens and other foreign matters. HDAC and Rac have already been demonstrated 
that they are required for efferocytosis [100]. Noda et al. reported that smoking could impair efferocytosis of neutrophils via inhibition of $\mathrm{HDAC} / \mathrm{Rac} / \mathrm{CD} 9$ pathways (Figure 2, c4). The following release of toxic intracellular contents from apoptotic neutrophils could cause tissue damage [100] (Figure 2, d).

The ability of ingesting respiratory pathogen is compromised in CSE-exposed neutrophils. As a result, it contributes to persistent existence of bacterium in the smokers' lungs and promotes further recruitment of neutrophils. Guzik et al. discovered a lack of apoptotic neutrophil populations in smokers' lungs, and these smokers exhibited an increased susceptibility to bacterial infections [111].

Human neutrophils share typical cell death features, including apoptosis, autophagy, and necrosis after exposure to CSE. These neutrophils could be effectively recognized and phagocytized by monoderived macrophages [111]. Neutrophils could also undergo a spontaneous and phagocytosisinduced apoptosis in a caspase-3-dependent manner. The suppression of caspase-3 activity induced by CSE does not alter spontaneous apoptosis but impair the phagocytic activity. The complex functions of caspase- 3 may contribute to the persistent existence of neutrophils in smokers' lungs. At the same time, caspase- 3 could also cause a higher incidence of community-acquired pneumonia [113]. In addition, percentage of sputum neutrophils undergoing spontaneous apoptosis in the subjects with COPD reduced significantly. The increased expression of both p50 and p65 subunits of NF-kappaB in neutrophils from COPD individuals may explain the phenomenon above [114] (Figure 2, c2). Interestingly, Makris et al. observed an increase of sputum apoptotic neutrophils' percentage in ex-smoking patients with COPD by the way of in situ detection with TUNEL assay technique [115]. Whether these conflict results are related with the distinct exclusion criteria of subjects needs further investigation.

3.4. Study on the Extracellular Traps of Neutrophils. Polymorphonuclear neutrophils have attracted new attention due to its ability of releasing web-like extracellular structures, which has been named as neutrophil extracellular traps (NETs) recently [116]. NETs' formation has been identified to be an essential part of innate immunity [117]. These NETs deriving from nuclear chromatin may have an ambiguous two-side effect on antimicrobial defense and host tissue damage. Microbicidal and cytotoxic proteins decorate the NETs, which are constituted with DNA strands of varying thickness. Their principal chemical structures have been characterized at molecular and ultrastructural levels in recent years. Nonetheless, many features relevant with cytotoxicity are still not clear completely [116]. Pedersen et al. observed a significant upregulation of NET formation, which was associated with significantly higher concentration of extracellular DNA in sputum supernatant of COPD [112]. Astrid et al. studied the genesis and structure of NETs from sputum of COPD patients. It was concluded that the genesis of NET was an integral part of COPD pathology. Moreover, the release of "beads-on-a-string" DNA studded with noncitrullinated histones, as Astrid et al. described, is a common feature of genesis of NETs in vivo. All of these are relevant to the antimicrobial and cytotoxic effects of NETs [116].

\subsection{Pharmacological Mechanism of Neutrophil Targeted} Therapy in COPD. Bronchodilators have always been a clinical first-line medication to ameliorate the symptoms in COPD [93]. Anderson et al. tested the effect of three $\beta 2$-agonists (formoterol, indacaterol, and salbutamol) on the inhibition to the proinflammatory activity. The results showed that formoterol and indacaterol could effectively decrease the potential harm produced by stimulated neutrophils in vitro, while the effect of salbutamol was weaker. The anti-inflammatory actions of formoterol and indacaterol could contribute to the therapeutics in COPD [118]. Tanabe et al. reported that thioredoxin-1 could improve neutrophilic inflammation by suppressing the release of GM-CSF and enhancing the expression of MAP kinase phosphatase 1. All these indicate that thioredoxin-1 is a novel potential therapeutic agent for treating the exacerbation of COPD [119]. Keratin sulfate disaccharide repeating unit designated L4 could significantly attenuate inflammation in the lung by reducing neutrophil influx as well as the levels of inflammatory cytokines, tissue-degrading enzymes (matrix metalloproteinases), and myeloperoxidase in BALF [120]. More clinical trials are needed to further reveal the potential of these related medicines.

Similar to asthma, ICSs are also the common therapy strategy in clinical practices to control COPD. However, the discontinuation of ICS can increase the inflammation in COPD showing as an increased sputum cell counts including neutrophils [121]. At the same time, the effect of ICS in COPD patients is also dependent on the genotype of glucocorticoid-induced transcript 1 rs37973 in COPD patients. The effect of dexamethasone-mediated neutrophil apoptosis is impaired in homozygous GG genotype. In addition, CSE-induced neutrophil apoptosis could only be slightly attenuated by a relatively higher concentration GG genotype. As for AA and AG genotypes, dexamethasone can reduce the proapoptotic effect of CSE in a concentration-dependent manner [122]. Besides, aminophylline and theophylline, two typical medicines used in therapy for COPD, could restore the impairment caused by trichostatin A (TSA), a Rac inhibitor. This protective effect may have the potential to develop a novel therapeutic strategy to restore efferocytosis in COPD patients [100].

\section{Neutrophils in Infectious Pneumonia}

Pneumonia is a common infectious lung disease accompanied with inflammation in airway and alveolar and interstitial lung. Pneumonia is usually categorized as communityacquired pneumonia (CAP) and hospital-acquired pneumonia (HAP). Bacterial infection is the most common etiology of pneumonia as observed in our clinical practice. Neutrophils can function with other immune cells synergistically to control the pathogenic infection in pneumonia [123]. Evidence on the participation of neutrophil in the pathogenesis of pneumonia has been accumulated in recent years. For example, the neutrophil percentage in BALF was higher in the 
relapse group of organizing pneumonias and may also be considered as a predictive factor of organizing pneumonia relapse as Onishi et al. reported [124]. But, on the contrary, CAP had significant low neutrophil counts in peripheral blood [125]. In addition, NLR together with red blood distribution can even be used as adjuncts to distinguish HAP and CAP [126].

\subsection{Pseudomonas aeruginosa Pneumonia. Pseudomonas} aeruginosa (PA) as an important opportunistic human pathogen can take part in the pathogenesis of pneumonia. PA lives in biofilm-like cell and aggregates at sites of chronic infection. During growth in a biofilm, $P$. aeruginosa dramatically increases the production of filamentous Pf bacteriophage (Pf phage). The Pf phage can trap within the lung PA by preventing the dissemination of $P$. aeruginosa from the lung in pneumonia and inhibit bacterial invasion of airway epithelial cultures. Importantly, the production of Pf phage was also associated with reduced neutrophil recruitment [127].

Mechanical ventilation is routinely used to treat patients with respiratory distress. However, a number of patients on ventilators exhibit enhanced susceptibility to infections and develop ventilator-associated pneumonia (VAP). PA is one of the most common species of bacteria found in these patients [128]. Mechanical ventilation after supernatant from PA-stimulated macrophages can induce more neutrophil sequestration in the lungs in wild-type mice than JNK1deficient mice. Moreover, the pathogenesis mechanism of PA-VAP may involve the production of TNF- $\alpha$ through activation of IKK/NF-kappaB pathways in alveolar macrophages and JNK signaling pathway in the lungs [129]. A recent research indicates that IRF-3 can exacerbate PA-induced mortality in mice by inhibiting neutrophil adhesion and recruitment to the lungs [130]. In addition, the inhibition of full-length receptor for advanced glycation end product (FL-RAGE) shedding can be a novel mechanism for controlling inflammation to acute PA pneumonia [131]. Deepening the exploration of its pathogenesis will contribute to the next future clinical application.

The persistent presence of PA and huge recruitment of neutrophils in the lung are associated with the elevated level of high mobility group box 1 (HMGB1) in airways in respiratory diseases [132]. Exposure to hyperoxia leads to a significant elevation in HMGB1 and increased mortality in C57BL/6 mice infected with PA. Treatment of these mice with a neutralizing anti-HMGB1 monoclonal antibody can result in a reduction in bacterial counts, injury, and numbers of neutrophils in the lungs and an increase in leukocyte phagocytic activity [133]. This finding reveals a potential medical target. More related clinical trials are needed to validate it.

4.2. Streptococcus pneumoniae Pneumonia. Streptococcus pneumoniae (SP) is a common cause of pneumonia and infective exacerbations of chronic lung disease [134]. During lung infection, mice colonized with SP had an increased early neutrophil recruitment and reduced bacterial colonyforming units in the lungs and BALF. Colonization-induced protection was lost when experiments were repeated in Bcell-deficient or neutrophil-deficient mice [134]. Yet how neutrophils specifically prevent SP lung infection has been complex and still unclear till now.

Nucleotide-binding oligomerization domain-containing (NOD) 2 as a pattern recognition receptor can detect peptidoglycan fragments of SP. Nod2-deficient blood neutrophils displayed a reduced capacity to internalize pneumococci in vitro. But NOD2 does not contribute to host defense during pneumococcal pneumonia. Pneumococcal capsule can impair recognition of SP by NOD2 as Hommes et al. discovered [135]. Triggering receptor expressed on myeloid cells-1 (TREM-1) is a receptor on phagocytes known to amplify TLR- and NOD-like receptor inflammatory signaling [136, 137]. TREM-1/3 deficiency leads to an increased lethality, accompanied by enhanced growth and dissemination of SP. Trem-1/3-deficient mice demonstrated a strongly impaired innate immune response in the airways reflecting as a delayed influx of neutrophils [138]. However, the influx of endothelial protein $\mathrm{C}$ receptor positive neutrophils into lung tissue can cause pneumonia after the infection of SP, because endothelial protein $\mathrm{C}$ receptor can impair antibacterial defense in pneumococcal pneumonia [139].

Moreover, IL-1 can contribute to the host defense against SP independent on the recruitment and the bacteria-killing ability of neutrophils [140]. Alveolar neutrophils with single immunoglobulin IL-1 receptor-related molecule (SIGIRR) deficiency exhibit an increased capacity to phagocytose viable pneumococci but no impact on neutrophil recruitment. Besides, SIGIRR as a negative regulator of TLR signaling can impair the antibacterial host defense during pneumonia caused by SP [141].

4.3. Staphylococcus aureus Pneumonia. Staphylococcus aureus (SA) is a Gram-positive bacterium that persistently colonizes about $20 \%$ of the human population [142]. This high prevalence of SA is responsible for various illnesses in humans and animals worldwide, including the respiratory diseases [143, 144]. Similar to SP, infection with SA can exhibit an early increase in neutrophils that did not persist despite continued presence of the bacteria in neonatal mice. However, adult mice exhibited an increase in neutrophil recruitment that coincided with reduced bacterial titers [145]. SA, as an important pathogen, can efficiently cleave the pulmonary surfactant protein-A (SP-A), a major component of immune functions during SA infections. This degradation appears to result in a decrease or complete abolishment of SP-A biological activity, including the promotion of SA phagocytosis by neutrophils [146]. Recently, Dietert et al. reported that in SA pneumonia murine model, the deficiency of calcium-activated chloride channel regulators (mCLCA3) can lead to a decrease of neutrophil infiltration during infection. Moreover, mCLCA3 appears mainly to modulate leukocyte response via IL-17 and murine CXCL-8 homologs in acute SA pneumonia [147].

4.4. Klebsiella pneumoniae Pneumonia. Nosocomial infection with Klebsiella pneumoniae (KP) is a frequent cause of gramnegative bacterial sepsis [148]. C-type lectin receptor is an 
innate immunity-related receptor, which can interact with pathogenic-associated molecular patterns [149]. Mincle and macrophage galactose-type lectin-1 (MGL1) are C-type lectin receptors. The deficiency of them can lead to a massive accumulation of neutrophils and a severe hyperinflammation in the lungs of KP-infected pneumonia. Importantly, Mincledeficient neutrophils had an impaired ability to phagocytize bacteria and to form extracellular traps (NETs), which could clear the invading KP [148]. Similarly, MGL1-deficient neutrophils exhibited an increased influx in pneumonic lungs of KP-infected mice. Neutrophilic inflammation resolution relies on MGL1 during KP infection [150]. As previously described, NLRC4 belongs to the NOD-like receptor family and is involved in the assembly of the inflammasome complex [151]. NLRC4 can participate in the neutrophil chemoattractant in the lungs infected by KP. NLRC4 signaling contributes to KP-induced lung inflammation and neutrophil accumulation, which can be partially rescued by exogenous IL-1 $\beta$ in the lungs of NLRC4-deficient mice [151]. Myeloid-related protein 8 (MRP8, S100A8) and MRP14 (S100A9) are the most abundant cytoplasmic proteins in neutrophils. MRP8/14 heterodimers can inhibit bacterial dissemination and prevent the growth of $\mathrm{KP}$ in vitro. Mrp14 can take part in the genesis of neutrophils NETs to inhibit KP growth. Taken together, MRP8/14 is a key player in protective innate immunity during KP pneumonia [152].

4.5. Mycoplasma pneumoniae Pneumonia and Recent Therapy Advances in Pneumonia. Mycoplasma pneumoniae is also a significant cause of respiratory diseases including CAP for all ages [153]. Mycoplasma pneumoniae pneumonia may be altered by the level of host cell-mediated immunity [154]. Mycoplasma pneumoniae pneumonia has an increase of neutrophils and IL-6, especially in severe group. Compared to acute stage, a decreased percentage of neutrophils and IL-6 level was observed at the recovery stage in children with severe Mycoplasma pneumoniae pneumonia [155]. However, treatment with prednisolone or cyclosporin-A leads to marked neutrophils and exudates in the alveolar lumen.

Some traditional antibiotic therapeutic strategies such as macrolide containing regimen showed no statistical difference between cytokine levels or neutrophil activity for CAP patient [156]. Targeted and precise therapy has made an advanced progress in recent years. Vaccine as a typical biological therapy strategy had been attracting a huge attention since it was created. For example, recombinant Bacillus Calmette-Guerin (BCG) vaccine can decrease the infiltration of neutrophils within airways and reduce the viral loads in BALF in mice infected with respiratory syncytial virus (RSV) showing a potential to prevent pneumonia [157]. Trivalent pneumococcal protein recombinant vaccine vaccination results in a reduction in SP-induced lethality, enhanced early clearance of SP in lungs due to more rapid and thorough phagocytosis of SP by neutrophils, and correspondingly, a reduction in lung inflammation and tissue damage [158]. In addition, cathelin-related antimicrobial peptide appears to be protective in models of pneumonia [159]. $\alpha$-Tocopherol form of vitamin $\mathrm{E}$ can reverse age- associated susceptibility to SP lung infection by decreasing pulmonary neutrophil recruitment [160].

\section{Neutrophils Participate in Other Respiratory Diseases}

5.1. Neutrophils in Lung Cancer. Lung cancer is still a common killer in all kinds of cancers and is also one of the most common cancers diagnosed globally [161]. The traditional role of neutrophils in pathogenesis of many kinds of tumors had already been reviewed by Zhang et al. briefly [162]. Here, we focus on the recent advances about the participation of neutrophils in lung cancer. It has been demonstrated that neutrophils could participate in the carcinogenesis in murine lung cancer model [163]. Lung squamous cell carcinoma mouse models contain more tumor-associated neutrophils compared to mouse adenocarcinomas [164]. Elastase from neutrophils could involve in lung cancer by inducing mitogenesis after entering the cells [165]. BALF from lung cancer patients contained higher level of neutrophils and lower percentage of total macrophages [166]. BALF of lung cancer patients had markedly higher levels of VEGF and IL-8, which was positively correlated to the numbers of neutrophils and lymphocytes. Tumor-associated neutrophils represent an important source of MMP-9, whose expression in tumor region is increased in non-small-cell lung cancer [167]. According to their results, the detection of infiltrating inflammatory cells and proangiogenic factors have the potential to be diagnosis indexes for cancerous inflammation in lungs [166].

Neutrophil-lymphocyte ratio (NLR), reflecting host immunity and systemic inflammation that facilitates tumor growth, could be an independent prognostic index for lung adenocarcinoma patients who undergo the complete resection [168]. NLR can also be an independent prognostic factor for overall survival. The evaluation of NLR can help identify patients with poor prognosis and appears to be a useful prognostic marker in clinical practices [169]. The model established by Jiang et al. utilizing multiple immunological markers, such as monocyte ratio, NLR, PD-L1 immunostaining score and PD-1-positive stained tumor-infiltrating lymphocyte counts, can offer a novel tool for survival prediction. This model has important clinical implications for patients with squamous non-small-cell lung cancer [170]. At the same time, NLR, together with other parameters such as age, gender, and smoking history, can be used to predict the prognosis of small cell lung cancer [171]. Consistent with the findings from Jeong et al. [172], Derman et al. found that the progressive increases of NLR are associated with the progressive disease, inferior overall survival, and weight loss in non-small-cell lung cancer patients [173]. The precise identification and prediction to the prognosis of all lung cancers with different histotypes is beneficial for future individualized therapy [170].

More recently, the role of myelomonocytic siglecs has attracted a greater attention $[174,175]$. It is a receptor engaging lineage with sialic acids with dual functions towards cancer progression depending on the different stages of tumor growth and the microenvironment. Neutrophils 
involved in this process play an important role. Neutrophils can express siglec-9 and interact with tumors. Tumors could interact and suppress the activation of neutrophils utilizing the ligand expressed on the tumor cells and acting on the siglec-9. In keeping with this, human polymorphism of the related gene that reduced siglec- 9 binding to carcinomas could improve the survival of patients with early non-smallcell lung cancer [176], which accounts for 85 percent of all lung cancer according to their histotypes [177]. As for lung cancer histotypes, it has been demonstrated that lung cancer could exhibit a pronounced heterogeneity and differential immunological characteristics. For example, adenosquamous carcinoma showed a histotype-specific recruitment of $\mathrm{CD}_{1} 1 \mathrm{~b}^{+} \mathrm{Gr}-1^{+}$tumor-associated neutrophils [178].

Metastases are the major cause of death from cancer. Lung is the most common metastatic site for many other cancers [179]. Impaired type I IFN signaling could develop more lung metastases. The higher metastasis is accompanied with massive neutrophil accumulation in the lungs. This is most likely due to elevated G-CSF levels in serum and enhanced CXCR2 expression on neutrophils. Reduced neutrophilic cytotoxicity against tumor cells can enhance metastasis [179]. Lung-infiltrating neutrophils facilitate an improved premetastatic niche formation [180-182]. Developing premetastatic niche can enhance metastasis [179]. In addition, intranasal delivery of CCL2 increases CD4+ T cell recruitment to the premetastatic niche of the lung, and this correlates with enhanced seeding and growth of tumor cells [183], while CCL2 shows a potential antitumor activity in tumor-entrained neutrophil-mediated tumor killing in vitro. In addition, $\gamma \delta$-T-cell could indirectly act on systemic expansion and polarization, suppress CD8+ T cell, and then cause a sequent metastasis formation. All of these indicate that the interaction between $\gamma \delta$-T-cell and neutrophil may contribute to the metastasis of carcinoma [184].

There are some interesting researches about how neutrophils participate into the pathogenesis of metastatic lung cancer. Bald et al. reported that ultraviolet (UV) exposure of primary cutaneous melanomas could promote metastasis dependent on the recruitment and activation of neutrophils which is initialed by HMGB1 [185]. It is also reported that an inhibitory host protein member of B7 family called as B7x may promote cancer cells to metastasize through interacting with innate and adaptive immune systems. The presence of $\mathrm{B} 7 \mathrm{x}$ is correlated with an increased infiltration of tumor-associated neutrophils into tumor-bearing lungs [186]. Colorectal cancer can process a lung metastasis, which is dependent on CCL15-CCR1 axis. Their immunofluorescent staining results showed that most CCR1+ cells around lung metastases were tumor-associated neutrophil [167]. Although the role of neutrophils in metastatic lung cancer is still unidentified clearly, more and more emerging researches will tell a systematic integral story in the future.

\subsection{Neutrophils in Pulmonary Fibrosis and Cystic Fibrosis.} Pulmonary fibrosis is a common interstitial lung disease [187]. Traditionally, pulmonary fibrosis is tightly associated with immune component in the lung. A greater number of neutrophils in the BALF were associated with the increased early mortality of pulmonary fibrosis [188]. The end-stage cystic fibrosis (CF) explant lung tissue showed an increase of neutrophils. At the same time, there was a disproportionate increase of neutrophils around the airway in CF [189].

Pulmonary fibrosis murine model is always established with bleomycin injection. Neutrophils can take part in the acute inflammatory process. The occurrence of the acute inflammation is accompanied with the production of collagen in parallel [190]. Interestingly, bleomycin-induced lung fibrosis can be relieved by the reduction of soluble glycosaminoglycan (GAG), which could reduce the neutrophil transmigration and decrease the CXCL-8/neutrophilmediated inflammation [191]. Carbohydrate antigen sialyl Lewis is secreted from the bronchial gland apically. Obayashi et al. reported that carbohydrate antigen sialyl Lewis in BALF could participate in the process of lung injury and repair in pulmonary fibrosis by modifying the function of neutrophils [192].

Serum amyloid $\mathrm{P}$ (SAP) is a pattern recognition molecule and could interact with pathogens and cell debris to promote their removal by macrophages and neutrophils [193]. Cox et al. reported that SAP could strongly affect several aspects of innate immune system and reduce fibrosis by binding to SAP-binding receptor (DC-SIGN), which is present on mouse lung epithelial cells. Binding of DC-SIGN receptor with SAP could reduce neutrophil accumulation in the acute lung inflammatory model and alleviate pulmonary fibrosis by increasing levels of immunosuppressant IL-10 [194]. In addition, they also reported that SAP could inhibit fibrocyte differentiation and reduce neutrophil adhesion by binding to $\mathrm{Fc}-\gamma$-RI on monocytes and binding to Fc- $\gamma$-RIIa on neutrophils, respectively [195].

Cystic fibrosis (CF) lung disease as a genetic disease is displayed as a chronic and nonresolving activation of innate immune system, accompanied with the release of neutrophilderived oxidants and proteases and chemokines and an infiltration of neutrophils into the airways [196]. Subjects with stable CF had not only significant elevated levels of proinflammatory genes and its products but also an elevated MMP8/9 and neutrophil elastase [197]. In addition, patients with CF and small airway disease had pronounced sputum neutrophil counts and elevated level of IL-6 [198]. The traditional role of immunity in CF had already been reviewed by Rieber et al. [196]. PMNs, which is recruited massively into the cystic fibrosis lumen, could modulate arginase 1 and suppress the early PMN-driven T cell in CF. All of these might hamper the resolution of infection and inflammation in $\mathrm{CF}$ airway lumen [199].

HMGB1 is an alarmin released from macrophages after infection or inflammation and is a biomarker of lung disease progression in patients with cystic fibrosis [200]. Entezari et al. demonstrated that the elevated levels of HMGB1 in $\mathrm{CF}$ airways were essential for neutrophil recruitment and persistence of PA in the lung, which could significantly contribute to mortality in cystic fibrosis [132]. The infection of PA can secrete epoxide hydrolase, a kind of CF transmembrane conductance regulator (CFTR) inhibitor, which could cause neutrophil activation and tissue inflammation. The hydrolase could also increase IL- 8 concentration, which 
drives neutrophils' transepithelial migration in vitro as illustrated above. Finally, the lung function of CF patients is impaired [201].

$\mathrm{CF}$ is a fatal recessive genetic disease. $\mathrm{Ng}$ et al. demonstrated that CF could be attributed to the mutations in the CFTR gene. In detail, the mutation of the gene could compromise the phagocytic capacity of neutrophils and contribute to the infection of the CF lung [202]. Recently, Duchesneau et al. found that bone marrow cell delivery therapy can contribute to the restoration of CFTR expression in airway epithelium by recruiting neutrophils and macrophages [203].

Moreover, other related gene polymorphisms or the difference of expressive level can also have effect on the pathogenesis of CF or other lung fibrotic diseases. Hector et al. demonstrated that interferon-related development regulator-1(IFRD1) expression of neutrophils was systemically upregulated in CF. This regulation was related to the production of ROS and was modulated by chemokines in airway fluids, such as CXCL-8 and CXCL-2. The decrease of lung function was associated with the genotype of IFRD1 [204]. Forkhead transcription factor 3 (Foxp3) is a critical regulator of Treg [205]. The overexpression of Foxp3 in radiationinduced lung inflammation also showed a significant inhibition of neutrophilic infiltration in BALF. At the same time, overexpression of Foxp3 can decrease the expression of inflammatory and fibrosis-related genes [206]. Extracellular superoxide dismutase 3 (SOD3) is the only extracellular enzymatic defense against the free radical, superoxide. Impaired SOD3 activity is implicated in inflammatory and fibrotic lung and vascular diseases as Mouradian et al. reviewed. However, the redistribution of superoxide dismutase 3 as a result of R213G single-nucleotide polymorphism could protect mice from bleomycin-induced fibrosis by resolving the neutrophil infiltration in BALF [207].

The potential therapy of pulmonary fibrosis is rarely reported in recent years. Recently, Yang et al. found that glaucocalyxin A (GLA) could exert antipulmonary fibrosis activity in mice. GLA could significantly improve survival in bleomycin-treated mice and reduce the weight loss caused by fibrosis. At the same time, GLA could alleviate the infiltration of neutrophils in the lungs and attenuate the increase of proinflammatory cytokines in lung tissue and BLAF. In addition, GLA could inhibit the activation of NF-kappaB in fibrotic lungs [208]. Acebilustat, as a potential leukotriene A4 hydrolase inhibitor, could reduce sputum neutrophil counts by $65 \%$ in CF patients treated with $100 \mathrm{mg}$ dosage [209]. Targeting chemotaxis of neutrophils has been a promising therapeutic direction [210]. Intracellular secretory leukoprotease inhibitor can exert an anti-inflammatory effect on neutrophils of individuals with CF and COPD by inhibiting the excessive influx of neutrophil [211]. PA401 as a recombinant therapeutic protein can disrupt the CXCL8:GAG complexes. And then, the chemokine CXCL8 is degraded. As a result, the chemotaxis of neutrophil and the inflammation decreased [212]. All in all, with the development of the detailed pathogenesis of pulmonary fibrosis, the targeted modulation of the related pathways may be of therapeutic benefit to patients.
5.3. Neutrophils in Bronchitis and Bronchiolitis. Acute and chronic bronchitis and bronchiolitis are common respiratory diseases [213]. Despite that most patients have a good prognosis, a few people still may be persistently unhealed. Immunity plays a critical but unidentified role in this process. The related inflammatory mechanism of neutrophils in airway inflammatory diseases and the role of proteases, mediators, and TLR2 in the incidence of the illness had already been reviewed [214]. But science has never stopped unraveling its nature. Recently, it has been demonstrated that neutrophilic infiltration in nasopharyngeal aspirate (NPA) samples was positively correlated with the degree of airway tissue injury in infants hospitalized with acute bronchiolitis [215].

Increased level of IL-8, a potent neutrophilic chemokine, is often strongly correlated with an increase in cellular infiltration [215]. For example, Dixon et al. reported that breastfed infants had lower level of IL-8 in their nasal compared to the formula-fed controls hospitalized with severe bronchiolitis. Meanwhile, there is a decrease in cellular infiltration, whose predominance is mature, secondary granuleladen neutrophils [216]. Chronic bronchitis is a risk factor for COPD. Patients with chronic bronchitis and COPD exhibit reduced immune regulation and increased innate immunity response in the lung [217]. Sahlander et al. have observed an increase of blood neutrophils in farmers exposed to the organic material. These farmers often suffered from the chronic bronchitis [218]. But in a more recent experiment, they only found that both the expression of CD62L and CD162 on blood neutrophils and the expression of CD14 on sputum neutrophils decreased. All of these indicated that chronic exposure of organic material may participate in the pathogenesis of chronic airway diseases, such as chronic bronchitis. This may involve the participation of neutrophils. In addition, they also discovered the exposure could increase the presence of bacteria in airways [219].

The presence of potentially pathogenic bacteria is positively correlated the severity of bronchiolitis. The percentage of neutrophils is higher in patients with potentially pathogenic bacteria [220]. Protracted bacterial bronchitis also had a marked neutrophil infiltration and more respiratory bacterial pathogens load, especially Haemophilus influenza. This may be related with activated innate immunity [221]. PA is very common in respiratory airways. Club cell secretory protein (CCSP) is a regulator, which could exert an immunosuppressive, anti-inflammatory, antiproteinase, and antiphospholipase A2 activities. Chronic PA inflammation can lead to chronic bronchitis in the CCSP-deficient mice. Neutrophils are increased in the BALF from the CCSPdeficient mice in comparison to wild-type mice [222]. In addition, colonization with Gram-negative bacteria was associated with higher levels of proinflammatory cytokines. The colonization would increase the severity of disease [223]. Borthwick et al. reported that conditioned media from PA-infected epithelial cells induced a potent proinflammatory phenotype in fibroblasts via an IL- $1 \alpha-/$ IL-1R-dependent signaling pathway. The evaluated level of IL- $1 \alpha$ is significantly correlated with IL- 8 and neutrophil percentage in BALF [224]. 
Viral etiology could potentially contribute to the pathogenesis of bronchiolitis [215]. Human respiratory syncytial virus (HRSV) infections have a close relationship with many respiratory diseases, such as bronchiolitis, asthma, and pneumonia [225]. Neutrophils together with its proteases, NETs, and cytotoxic and direct interaction with infected epithelial cell play an important role in the pathological process [225]. RSV could induce NET formation in vitro. Conversely, NETs can capture RSV virions, indicating an antiviral role [226]. Bronchiolitis, usually requiring hospitalization in infants, is caused predominantly by RSV. It is one of the main causes of infant mortality and morbidity in developed world [216]. Suarez et al. reported that infants with RSV bronchiolitis had more systemic inflammatory cells, such as neutrophils and leukocytes, and more pathogenic bacterial colonization in nasopharyngeal. Besides, neutrophil infiltration is independent on the viral etiology nor the degree of viral coinfection, providing support for the neutrophil as a target of therapeutic intervention for the treatment of bronchiolitis in all virus-positive infants as Cavallaro et al. reported [215]. It is pleasuring that BPZE1, as a kind of attenuated Bordetella pertussis vaccine, could markedly attenuate RSV by inducing the efflux of neutrophils and increasing the production of IL-17 by CD4+ T cells [227].

In a special case, patients undergoing lung transplantation often suffer from obliterative bronchiolitis (OB). Clinical research showed us that the percentage of lymphocytes and neutrophils increased and the percentage of macrophages reduced in BALF of patients with OB [228]. This is similar with the reports, which suggest an involvement of neutrophils in $\mathrm{OB}$ from both Vandermeulen et al. and Eckrich et al. [229, 230]. Tiriveedhi et al. reported that neutrophil can participate in the obliterative airway disease and cause the early injury after passive transfer of CD8+ T cells [231]. They also reported that antibodies to MHC class I of the transplanted lung could induce both innate and adaptive cellular immune responses, which is characterized by a predominance of Th17. Their data indicated that Treg cells could suppress "anti-MHC induced IL-8-mediated neutrophil infiltration," which is critical for the development of obliterative airway disease [232]. VEGF-C/VEGFR-3 signaling can be involved in the pathogenesis of $\mathrm{OB}$ [233]. Upregulation of VEGF-C/VEGFR-3 signaling could induce epithelial activation, neutrophil chemotaxis, and significant neutrophilia. Both neutrophils and neutrophil chemoattractant human IL-8 contribute to the development of OB for its inflammatory infiltration [233].

Hyaluronan is an extracellular matrix component, which has been demonstrated to activate innate immunity, regulate inflammation, and could accumulate in BALF and blood of lung transplant recipients with $\mathrm{OB}$. The low-molecularweight form of hyaluronan can abolish the tolerance and promote the rejection of lung transplant through a mechanism dependent on innate immunity and neutrophils [234]. More details about its mechanism need further illustration. Adenosine is produced to protect tissues from injury when ischemia or inflammation occurs [235]. Zhao et al. described the role of $\mathrm{A}_{2 \mathrm{~B}} \mathrm{R}$, a receptor of adenosine in the pathogenesis of OB. Neutrophil infiltration was decreased in $A_{2 B} R$ knock- out OB model on day 3 and day 21 but increased in wild-type models in the same time point. The results showed us that neutrophils could also take part in the pathogenesis of $\mathrm{OB}$ depending on $\mathrm{A}_{2 \mathrm{~B}} \mathrm{R}$ to some extent [235]. In contrast, $\mathrm{A}_{2 \mathrm{~A}} \mathrm{R}$ could also participate in the inflammatory process in $\mathrm{OB}$. Neutrophil infiltration was increased in $\mathrm{A}_{2 \mathrm{~A}} \mathrm{R}$ knock-out OB model [236]. Whether the exogenous pharmaceuticals of adenosine can be helpful to relieving $\mathrm{OB}$ needs future clinical validation.

\section{Conclusions}

The critical role of neutrophils in immunity-associated diseases including respiratory diseases cannot be overlooked. The colonization of microbiota in airway acts as a trigger of neutrophilic inflammation. Different stimulations from our environment could produce a chemotactic activity towards the inflammatory sites for neutrophils. Innate and adaptive immune component could participate in the activation of neutrophils in many different respiratory diseases. Neutrophils often cooperate with lymphocytes synergistically and constitute a huge immune regulatory network. Different PRRs, such as TLR and NLR families, are indispensable to interact with DAMPs from the dying cells. After the immunological mission of neutrophils is accomplished, the apoptosis of neutrophils is very important for the withdrawal of inflammation or tissue damage. But the prosurvival of neutrophils usually aggravates the injury. Proteases, as key functional component from neutrophils, could exert a double-sided effect on the pathogenesis of respiratory diseases. They could clear the adverse factors to maintain the homoeostasis. On the other hand, their excessive secretion also contributes the injury of normal tissue. In addition, neutrophils have developed a unique NET system to play their role of double-edged sword. It can not only trap the pathogens but also amplify the inflammatory cascade. However, there are still many unanswered questions on the role of neutrophils in lung cancer, pulmonary fibrosis, cystic fibrosis, bronchitis, and bronchiolitis. Despite that the detailed pathogeneses of these diseases are fragments for now, it is believed that more and more scientific researches will connect them. Different pharmaceutical intervention had been investigated to act upon the neutrophils and manifested a promising effect to some extent. The clarification on the mechanism of traditional drugs and the development of new precise medicine, such as monoantibody and vaccine will be the following research direction. Neutrophils have the potential to be a new therapeutic target as expected in the future. Finally, understanding how neutrophils cooperate with other immune component to integrate the disease pathogenic mechanisms, and exploring how to develop novel avenues for therapeutic strategies aimed at the key pathway involved of neutrophils, will offer further insights and inform better treatment of respiratory diseases.

\section{Conflicts of Interest}

The authors all declare that there is no any conflict of interests regarding the publication of this review. 


\section{Acknowledgments}

The authors are thankful for the encouragement and inspiration from their project investigator team. All the researches in the authors' laboratory are supported by Science and Technology Development Plan of Jilin Province of China (Grant no. 20150519015JH).

\section{References}

[1] K. F. Chung, "Targeting the interleukin pathway in the treatment of asthma," Lancet, vol. 386, no. 9998, pp. 1086-1096, 2015.

[2] A. Jemal, F. Bray, M. M. Center, J. Ferlay, E. Ward, and D. Forman, "Global cancer statistics," CA: A Cancer Journal for Clinicians, vol. 61, no. 2, pp. 69-90, 2011.

[3] P. Burney, S. Suissa, J. B. Soriano et al., "The pharmacoepidemiology of COPD: recent advances and methodological discussion," The European Respiratory Journal. Supplement, vol. 43, no. 43, pp. 1s-44s, 2003.

[4] S. T. Gohy, C. Hupin, C. Pilette, and M. Z. Ladjemi, "Chronic inflammatory airway diseases: The central role of the epithelium revisited," Clinical and Experimental Allergy, vol. 46, no. 4, pp. 529-542, 2016.

[5] J. G. Bartlett, S. F. Dowell, L. A. Mandell, T. M. File Jr, D. M. Musher, and M. J. Fine, "Practice guidelines for the management of community-acquired pneumonia in adults. Infectious Diseases Society of America," Clinical Infectious Diseases, vol. 31, no. 2, pp. 347-382, 2000.

[6] M. Ekstrom and A. Bornefalk-Hermansson, "Cardiovascular and antacid treatment and mortality in oxygen-dependent pulmonary fibrosis: A population-based longitudinal study," Respirology, vol. 21, no. 4, pp. 705-711, 2016.

[7] A. M. Mangi, V. Bansal, G. Li, M. S. Pieper, O. Gajic, and E. Festic, "Pre-hospital use of inhaled corticosteroids and inhaled beta agonists and incidence of ARDS: a populationbased study," Acta Medica Academica, vol. 44, no. 2, pp. 109-116, 2015.

[8] S. Masieri, C. Cavaliere, E. Begvarfaj, D. Rosati, and A. Minni, "Effects of omalizumab therapy on allergic rhinitis: a pilot study," European Review for Medical and Pharmacological Sciences, vol. 20, no. 24, pp. 5249-5255, 2016.

[9] X. F. Xu, H. P. Dai, Y. M. Li, F. Xiao, and C. Wang, "Mass spectrometry-based proteomics in acute respiratory distress syndrome: a powerful modality for pulmonary precision medicine," Chinese Medical Journal, vol. 129, no. 19, pp. 2357-2364, 2016.

[10] V. Perri, E. Gianchecchi, R. Scarpa et al., "Altered B cell homeostasis and Toll-like receptor 9-driven response in patients affected by autoimmune polyglandular syndrome type 1: Altered B cell phenotype and dysregulation of the B cell function in APECED patients," Immunobiology, vol. 222, no. 2, pp. 372-383, 2017.

[11] C. Cardamone, R. Parente, G. D. Feo, and M. Triggiani, "Mast cells as effector cells of innate immunity and regulators of adaptive immunity," Immunology Letters, vol. 178, pp. 10-14, 2016.

[12] J. J. Marchalonis, S. Kaveri, S. Lacroix-Desmazes, and M. D. Kazatchkine, "Natural recognition repertoire and the evolutionary emergence of the combinatorial immune system," The FASEB Journal, vol. 16, no. 8, pp. 842-8, 2002.
[13] L. Ginaldi, M. De Martinis, A. D'Ostilio, L. Marini, M. F. Loreto, and D. Quaglino, "The immune system in the elderly: III. Innate immunity," Immunologic Research, vol. 20, no. 2, pp. 117-126, 1999.

[14] H. Li, T. Yang, F. Y. Li, Q. Ning, and Z. M. Sun, "TLR4 Overexpression inhibits endothelial PAS domaincontaining protein 1 expression in the lower respiratory tract of patients with chronic COPD," Cellular Physiology and Biochemistry, vol. 39, no. 2, pp. 685-692, 2016.

[15] K. W. Chen, J. S. Bezbradica, C. J. Gross et al., "The murine neutrophil NLRP3 inflammasome is activated by soluble but not particulate or crystalline agonists," European Journal of Immunology, vol. 46, no. 4, pp. 10041010, 2016.

[16] Y. Ren, L. Hua, X. Meng et al., "Correlation of surface Toll-like receptor 9 expression with IL-17 production in neutrophils during septic peritonitis in mice induced by E. coli," Mediators of Inflammation, vol. 2016, Article ID 3296307, 17 pages, 2016.

[17] J. J. Eastman, K. J. Cavagnero, A. S. Deconde et al., "Group 2 innate lymphoid cells are recruited to the nasal mucosa in patients with aspirin-exacerbated respiratory disease," The Journal of Allergy and Clinical Immunology, 2017, [Epub ahead of print].

[18] D. L. Riegert-Johnson, J. A. Godfrey, J. L. Myers, R. D. Hubmayr, W. J. Sandborn, and E. V. Loftus Jr, "Delayed hypersensitivity reaction and acute respiratory distress syndrome following infliximab infusion," Inflammatory Bowel Diseases, vol. 8, no. 3, pp. 186-191, 2002.

[19] T. A. Gheita, H. A. Gheita, and S. A. Kenawy, "Rituximab restored the muscle power and rescued from a refractory fatal respiratory failure in a patient with elderly-onset polymyositis," Joint, Bone, Spine, vol. 79, no. 1, pp. 101102, 2012.

[20] F. M. Konrad, S. Braun, K. C. Ngamsri, I. Vollmer, and J. Reutershan, "Heme oxygenase-1 attenuates acute pulmonary inflammation by decreasing the release of segmented neutrophils from the bone marrow," American Journal of Physiology. Lung Cellular and Molecular Physiology, vol. 307, no. 9, pp. L707-L717, 2014.

[21] C. Rosales, N. Demaurex, C. A. Lowell, and E. Uribe-Querol, "Neutrophils: Their role in innate and adaptive immunity," Journal of Immunology Research, vol. 2016, Article ID 1469780, 2 pages, 2016.

[22] W. Liu, S. Liu, M. Verma et al., "Mechanism of TH2/TH17predominant and neutrophilic TH2/TH17-low subtypes of asthma," The Journal of Allergy and Clinical Immunology, 2016, [Epub ahead of print].

[23] C. J. Gordon, P. M. Phillips, A. Ledbetter et al., "Active vs. sedentary lifestyle from weaning to adulthood and susceptibility to ozone in rats," American Journal of Physiology. Lung Cellular and Molecular Physiology, vol. 312, no. 1, pp. L100-L109, 2017.

[24] A. Vargas, F. Roux-Dalvai, A. Droit, and J. P. Lavoie, "Neutrophil-derived exosomes: a new mechanism contributing to airway smooth muscle remodeling," American Journal of Respiratory Cell and Molecular Biology, vol. 55, no. 3, pp. 450-461, 2016.

[25] D. L. Pham, G. Y. Ban, S. H. Kim et al., "Neutrophil autophagy and extracellular DNA traps contribute to airway inflammation in severe asthma," Clinical and Experimental Allergy, vol. 47, no. 1, pp. 57-70, 2017. 
[26] Global Initiative for Asthma, Global Strategy for Asthma Management and Prevention, GINA, Fontana, WI, USA, 2017, http://ginasthma.org/download/317/.

[27] F. Wang, X. Y. He, K. J. Baines et al., "Different inflammatory phenotypes in adults and children with acute asthma," The European Respiratory Journal, vol. 38, no. 3, pp. 567574, 2011.

[28] D. B. Khatry, D. L. Gossage, G. P. Geba et al., "Discriminating sputum-eosinophilic asthma: accuracy of cutoffs in blood eosinophil measurements versus a composite index, ELEN," The Journal of Allergy and Clinical Immunology, vol. 136, no. 3, pp. 812-814 e2, 2015.

[29] J. L. Simpson, R. Scott, M. J. Boyle, and P. G. Gibson, "Inflammatory subtypes in asthma: assessment and identification using induced sputum," Respirology, vol. 11, no. 1, pp. 54-61, 2006.

[30] M. Kupczyk, B. Dahlen, P. J. Sterk et al., "Stability of phenotypes defined by physiological variables and biomarkers in adults with asthma," Allergy, vol. 69, no. 9, pp. 11981204, 2014.

[31] L. Fleming, L. Tsartsali, N. Wilson, N. Regamey, and A. Bush, "Sputum inflammatory phenotypes are not stable in children with asthma," Thorax, vol. 67, no. 8, pp. 675-681, 2012.

[32] M. T. Al-Samri, A. Benedetti, D. Prefontaine et al., "Variability of sputum inflammatory cells in asthmatic patients receiving corticosteroid therapy: a prospective study using multiple samples," The Journal of Allergy and Clinical Immunology, vol. 125, no. 5, pp. 1161-1163.e4, 2010.

[33] M. Wang, P. Gao, X. Wu et al., "Impaired anti-inflammatory action of glucocorticoid in neutrophil from patients with steroid-resistant asthma," Respiratory Research, vol. 17, no. 1, p. 153, 2016.

[34] E. Collisson, L. Griggs, and Y. Drechsler, "Macrophages from disease resistant B2 haplotype chickens activate $\mathrm{T}$ lymphocytes more effectively than macrophages from disease susceptible B19 birds," Developmental and Comparative Immunology, vol. 67, pp. 249-256, 2017.

[35] S. Vedel-Krogh, S. Fallgaard Nielsen, P. Lange, J. Vestbo, and B. G. Nordestgaard, "Association of blood eosinophil and blood neutrophil counts with asthma exacerbations in the Copenhagen general population study," Clinical Chemistry, vol. 63, no. 4, pp. 823-832, 2017.

[36] E. M. Uribe Echevarria, C. A. Maldonado, A. M. Uribe Echevarria, and A. Aoki, "Neutrophil predominance in induced sputum from asthmatic patients. Therapeutic implications and role of clara cell $16-\mathrm{KD}$ protein," Medicina, vol. 71, no. 4, pp. 343-349, 2011.

[37] A. Beigelman, G. M. Weinstock, and L. B. Bacharier, "The relationships between environmental bacterial exposure, airway bacterial colonization, and asthma," Current Opinion in Allergy and Clinical Immunology, vol. 14, no. 2, pp. 137-142, 2014.

[38] B. J. Green, S. Wiriyachaiporn, C. Grainge et al., "Potentially pathogenic airway bacteria and neutrophilic inflammation in treatment resistant severe asthma," PloS One, vol. 9, no. 6, article e100645, 2014.

[39] A. T. Essilfie, J. L. Simpson, J. C. Horvat et al., "Haemophilus influenzae infection drives IL-17-mediated neutrophilic allergic airways disease," PLoS Pathogens, vol. 7, no. 10, article e1002244, 2011.
[40] R. Alam, J. Good, D. Rollins et al., "Airway and serum biochemical correlates of refractory neutrophilic asthma," The Journal of Allergy and Clinical Immunology, 2017, [Epub ahead of print].

[41] S. Hadebe, F. Kirstein, K. Fierens et al., "Microbial ligand costimulation drives neutrophilic steroid-refractory asthma," PloS One, vol. 10, no. 8, article e0134219, 2015.

[42] T. Ito, K. Hirose, A. Norimoto et al., "Dectin-1 plays an important role in house dust mite-induced allergic airway inflammation through the activation of $\mathrm{CD} 1 \mathrm{~b}+$ dendritic cells," Journal of Immunology, vol. 198, no. 1, pp. 61-70, 2017.

[43] T. Mosca, M. C. Menezes, A. V. Silva, R. Stirbulov, and W. C. Forte, "Chemotactic and phagocytic activity of blood neutrophils in allergic asthma," Immunological Investigations, vol. 44, no. 5, pp. 509-520, 2015.

[44] S. Lavinskiene, J. Jeroch, K. Malakauskas, I. Bajoriuniene, J. Jackute, and R. Sakalauskas, "Peripheral blood neutrophil activity during Dermatophagoides pteronyssinus-induced late-phase airway inflammation in patients with allergic rhinitis and asthma," Inflammation, vol. 35, no. 4, pp. 1600 1609, 2012.

[45] L. Q. Siew, S. Y. Wu, S. Ying, and C. J. Corrigan, "Cigarette smoking increases bronchial mucosal IL-17A expression in asthmatics, which acts in concert with environmental aeroallergens to engender neutrophilic inflammation," Clinical and Experimental Allergy, 2017, [Epub ahead of print].

[46] Y. Kobayashi, H. Wada, C. Rossios et al., "A novel macrolide/ fluoroketolide, solithromycin (CEM-101), reverses corticosteroid insensitivity via phosphoinositide 3-kinase pathway inhibition," British Journal of Pharmacology, vol. 169, no. 5, pp. 1024-1034, 2013.

[47] D. C. Newcomb, U. S. Sajjan, D. R. Nagarkar et al., "Human rhinovirus $1 \mathrm{~B}$ exposure induces phosphatidylinositol 3kinase-dependent airway inflammation in mice," American Journal of Respiratory and Critical Care Medicine, vol. 177, no. 10, pp. 1111-1121, 2008.

[48] X. Xu, N. Gera, H. Li et al., "GPCR-mediated PLCbetagamma/PKCbeta/PKD signaling pathway regulates the cofilin phosphatase slingshot 2 in neutrophil chemotaxis," Molecular Biology of the Cell, vol. 26, no. 5, pp. 874-886, 2015.

[49] J. Doukas, L. Eide, K. Stebbins et al., “Aerosolized phosphoinositide 3-kinase gamma/delta inhibitor TG100-115 [3-[2,4diamino-6-(3-hydroxyphenyl)pteridin-7-yl]phenol] as a therapeutic candidate for asthma and chronic obstructive pulmonary disease," The Journal of Pharmacology and Experimental Therapeutics, vol. 328, no. 3, pp. 758-765, 2009.

[50] T. M. Randis, K. D. Puri, H. Zhou, and T. G. Diacovo, "Role of PI3Kdelta and PI3Kgamma in inflammatory arthritis and tissue localization of neutrophils," European Journal of Immunology, vol. 38, no. 5, pp. 1215-1224, 2008.

[51] W. P. Fung-Leung, "Phosphoinositide 3-kinase delta (PI3Kdelta) in leukocyte signaling and function," Cellular Signalling, vol. 23, no. 4, pp. 603-608, 2011.

[52] M. Thomas, M. J. Edwards, E. Sawicka et al., "Essential role of phosphoinositide 3-kinase gamma in eosinophil chemotaxis within acute pulmonary inflammation," Immunology, vol. 126, no. 3, pp. 413-422, 2009.

[53] S. Sriskantharajah, N. Hamblin, S. Worsley, A. R. Calver, E. M. Hessel, and A. Amour, "Targeting phosphoinositide 3kinase delta for the treatment of respiratory diseases," Annals 
of the new York Academy of Sciences, vol. 1280, no. 1, pp. 3539, 2013.

[54] R. Y. Kim, J. C. Horvat, J. W. Pinkerton et al., "MicroRNA-21 drives severe, steroid-insensitive experimental asthma by amplifying phosphoinositide 3-kinase-mediated suppression of histone deacetylase 2," The Journal of Allergy and Clinical Immunology, vol. 139, no. 2, pp. 519-532, 2017.

[55] V. Gupta, A. Khan, A. Higham et al., "The effect of phosphatidylinositol-3 kinase inhibition on matrix metalloproteinase- 9 and reactive oxygen species release from chronic obstructive pulmonary disease neutrophils," International Immunopharmacology, vol. 35, pp. 155-162, 2016.

[56] J. Milara, J. Lluch, P. Almudever, J. Freire, Q. Xiaozhong, and J. Cortijo, "Roflumilast N-oxide reverses corticosteroid resistance in neutrophils from patients with chronic obstructive pulmonary disease," The Journal of Allergy and Clinical Immunology, vol. 134, no. 2, pp. 314-322, 2014.

[57] Z. Dong, L. Xiong, W. Zhang et al., "Holding the inflammatory system in check: TLRs and their targeted therapy in asthma," Mediators of Inflammation, vol. 2016, Article ID 2180417, 8 pages, 2016.

[58] B. J. Hsia, G. S. Whitehead, S. Y. Thomas et al., "Trif-dependent induction of Th17 immunity by lung dendritic cells," Mucosal Immunology, vol. 8, no. 1, pp. 186-197, 2015.

[59] J. Zhu, S. D. Message, Y. Qiu et al., "Airway inflammation and illness severity in response to experimental rhinovirus infection in asthma," Chest, vol. 145, no. 6, pp. 1219-1229, 2014.

[60] F. S. Tang, D. Van Ly, K. Spann et al., "Differential neutrophil activation in viral infections: enhanced TLR-7/8-mediated CXCL8 release in asthma," Respirology, vol. 21, no. 1, pp. 172-179, 2016.

[61] K. Hosoki, S. Ying, C. Corrigan et al., "Analysis of a panel of 48 cytokines in BAL fluids specifically identifies IL-8 levels as the only cytokine that distinguishes controlled asthma from uncontrolled asthma, and correlates inversely with FEV1," PloS One, vol. 10, no. 5, article e0126035, 2015.

[62] M. A. Panaro and V. Mitolo, "Cellular responses to FMLP challenging: a mini-review," Immunopharmacology and Immunotoxicology, vol. 21, no. 3, pp. 397-419, 1999.

[63] F. S. Tang, G. J. Foxley, P. G. Gibson, J. K. Burgess, K. J. Baines, and B. G. Oliver, "Altered innate immune responses in neutrophils from patients with well- and suboptimally controlled asthma," Mediators of Inflammation, vol. 2015, Article ID 219374, 11 pages, 2015.

[64] K. Page, K. M. Lierl, V. S. Hughes, P. Zhou, J. R. Ledford, and M. Wills-Karp, "TLR2-mediated activation of neutrophils in response to German cockroach frass," Journal of Immunology, vol. 180, no. 9, pp. 6317-6324, 2008.

[65] K. Hosoki, T. Itazawa, I. Boldogh, and S. Sur, "Neutrophil recruitment by allergens contribute to allergic sensitization and allergic inflammation," Current Opinion in Allergy and Clinical Immunology, vol. 16, no. 1, pp. 45-50, 2016.

[66] J. W. McAlees, G. S. Whitehead, I. T. Harley et al., "Distinct Tlr4-expressing cell compartments control neutrophilic and eosinophilic airway inflammation," Mucosal Immunology, vol. 8, no. 4, pp. 863-873, 2015.

[67] L. Uller, C. G. Persson, and J. S. Erjefalt, "Resolution of airway disease: Removal of inflammatory cells through apoptosis, egression or both?" Trends in Pharmacological Sciences, vol. 27, no. 9, pp. 461-466, 2006.
[68] K. J. Baines, J. L. Simpson, R. J. Scott, and P. G. Gibson, "Immune responses of airway neutrophils are impaired in asthma," Experimental Lung Research, vol. 35, no. 7, pp. 554-569, 2009.

[69] K. J. Baines, J. L. Simpson, N. A. Bowden, R. J. Scott, and P. G. Gibson, "Differential gene expression and cytokine production from neutrophils in asthma phenotypes," The European Respiratory Journal, vol. 35, no. 3, pp. 522-531, 2010.

[70] J. L. Simpson, H. Powell, M. J. Boyle, R. J. Scott, and P. G. Gibson, "Clarithromycin targets neutrophilic airway inflammation in refractory asthma," American Journal of Respiratory and Critical Care Medicine, vol. 177, no. 2, pp. 148-155, 2008.

[71] K. Maneechotesuwan, S. Essilfie-Quaye, S. A. Kharitonov, I. M. Adcock, and P. J. Barnes, "Loss of control of asthma following inhaled corticosteroid withdrawal is associated with increased sputum interleukin- 8 and neutrophils," Chest, vol. 132, no. 1, pp. 98-105, 2007.

[72] P. Katavolos, C. A. Ackerley, M. E. Clark, and D. Bienzle, "Clara cell secretory protein increases phagocytic and decreases oxidative activity of neutrophils," Veterinary Immunology and Immunopathology, vol. 139, no. 1, pp. 1-9, 2011.

[73] O. Cote, M. E. Clark, L. Viel et al., "Secretoglobin 1A1 and $1 \mathrm{~A} 1 \mathrm{~A}$ differentially regulate neutrophil reactive oxygen species production, phagocytosis and extracellular trap formation," PloS One, vol. 9, no. 4, article e96217, 2014.

[74] B. P. Tian, L. X. Xia, Z. Q. Bao et al., "Bcl-2 inhibitors reduce steroid-insensitive airway inflammation," The Journal of Allergy and Clinical Immunology, 2016, [Epub ahead of print].

[75] M. Uddin, G. Nong, J. Ward et al., "Prosurvival activity for airway neutrophils in severe asthma," Thorax, vol. 65, no. 8, pp. 684-689, 2010.

[76] D. H. Kim, E. Choi, J. S. Lee et al., "House dust mite allergen regulates constitutive apoptosis of normal and asthmatic neutrophils via Toll-like receptor 4," PloS One, vol. 10, no. 5, article e0125983, 2015.

[77] N. R. Lee, S. Y. Baek, A. Gu et al., "House dust mite allergen suppresses neutrophil apoptosis by cytokine release via PAR2 in normal and allergic lymphocytes," Immunologic Research, vol. 64, no. 1, pp. 123-132, 2016.

[78] L. I. Sikkeland, H. L. Johnsen, T. B. Riste et al., "Sputum neutrophils are elevated in smelter workers, and systemic neutrophils are associated with rapid decline in FEV1," Occupational and Environmental Medicine, vol. 73, no. 7, pp. 459-466, 2016.

[79] P. L. Bruijnzeel, M. Uddin, and L. Koenderman, "Targeting neutrophilic inflammation in severe neutrophilic asthma: can we target the disease-relevant neutrophil phenotype?" Journal of Leukocyte Biology, vol. 98, no. 4, pp. 549-556, 2015.

[80] H. A. Murad, H. S. Habib, M. M. Rafeeq, M. I. Sulaiman, A. S. Abdulrahman, and M. N. Khabaz, "Co-inhalation of roflumilast, rather than formoterol, with fluticasone more effectively improves asthma in asthmatic mice," Experimental Biology and Medicine (Maywood, N.J.), vol. 242, no. 5, pp. 516-526, 2017.

[81] W. Han, J. Li, H. Tang, and L. Sun, "Treatment of obese asthma in a mouse model by simvastatin is associated with improving dyslipidemia and decreasing leptin level," Biochemical and Biophysical Research Communications, vol. 484, no. 2, pp. 396-402, 2017. 
[82] C. Borlone, N. Morales, C. Henriquez et al., "In vitro effects of tamoxifen on equine neutrophils," Research in Veterinary Science, vol. 110, pp. 60-64, 2017.

[83] J. H. Yu, L. Long, Z. X. Luo, and J. R. You, "Effect of PPARgamma agonist (rosiglitazone) on the secretion of Th2 cytokine in asthma mice," Asian Pacific Journal of Tropical Medicine, vol. 10, no. 1, pp. 64-68, 2017.

[84] S. Jurcevic, C. Humfrey, M. Uddin, S. Warrington, B. Larsson, and C. Keen, "The effect of a selective CXCR2 antagonist (AZD5069) on human blood neutrophil count and innate immune functions," British Journal of Clinical Pharmacology, vol. 80, no. 6, pp. 1324-1336, 2015.

[85] Y. Wei, J. Liu, H. Zhang et al., "Ligustrazine attenuates inflammation and the associated chemokines and receptors in ovalbumine-induced mouse asthma model," Environmental Toxicology and Pharmacology, vol. 46, pp. 55-61, 2016.

[86] C. J. Liou, Y. L. Huang, W. C. Huang, K. W. Yeh, T. Y. Huang, and C. F. Lin, "Water extract of Helminthostachys zeylanica attenuates LPS-induced acute lung injury in mice by modulating NF-kappaB and MAPK pathways," Journal of Ethnopharmacology, vol. 199, pp. 30-38, 2017.

[87] Y. H. Kim, Y. J. Choi, M. K. Kang et al., "Astragalin inhibits allergic inflammation and airway thickening in ovalbumin-challenged mice," Journal of Agricultural and Food Chemistry, vol. 65, no. 4, pp. 836-845, 2017.

[88] D. A. de Almeida, S. I. Rosa, T. C. da Cruz et al., "Mandevilla Longiflora (Desf.) Pichon improves airway inflammation in a murine model of allergic asthma," Journal of Ethnopharmacology, vol. 200, pp. 51-59, 2017.

[89] Z. Zhakeer, M. Hadeer, Z. Tuerxun, and K. Tuerxun, "Bufalin inhibits the inflammatory effects in asthmatic mice through the suppression of nuclear factor-kappa B activity," Pharmacology, vol. 99, no. 3-4, pp. 179-187, 2017.

[90] X. Fei, X. Zhang, G. Q. Zhang et al., "Cordycepin inhibits airway remodeling in a rat model of chronic asthma," Biomedicine \& Pharmacotherapy, vol. 88, pp. 335-341, 2017.

[91] J. D. de Boer, M. Berger, C. J. Majoor et al., "Activated protein $\mathrm{C}$ inhibits neutrophil migration in allergic asthma: a randomised trial," The European Respiratory Journal, vol. 46, no. 6, pp. 1636-1644, 2015.

[92] Y. Liu, H. Zhang, R. Ni, W. Q. Jia, and Y. Y. Wang, "IL-4R suppresses airway inflammation in bronchial asthma by inhibiting the IL-4/STAT6 pathway," Pulmonary Pharmacology \& Therapeutics, vol. 43, pp. 32-38, 2017.

[93] M. Meijer, G. T. Rijkers, and F. J. van Overveld, "Neutrophils and emerging targets for treatment in chronic obstructive pulmonary disease," Expert Review of Clinical Immunology, vol. 9, no. 11, pp. 1055-1068, 2013.

[94] R. Farah, R. Ibrahim, M. Nassar, D. Najib, Y. Zivony, and E. Eshel, "Neutrophil lymphocyte ratio better addition to CRP than CD64 index as marker for infection in COPD," Panminerva Medica, 2017, [Epub ahead of print].

[95] A. Guillon, Y. Jouan, D. Brea et al., "Neutrophil proteases alter the interleukin-22-receptor-dependent lung antimicrobial defence," The European Respiratory Journal, vol. 46, no. 3, pp. 771-782, 2015.

[96] D. L. Bratton and P. M. Henson, "Neutrophil clearance: when the party is over, clean-up begins," Trends in Immunology, vol. 32, no. 8, pp. 350-357, 2011.

[97] R. Siva, M. Bafadhel, W. Monteiro, C. E. Brightling, and I. D. Pavord, "Effect of levofloxacin on neutrophilic airway inflammation in stable COPD: a randomized, double-blind, placebo-controlled trial," International Journal of Chronic Obstructive Pulmonary Disease, vol. 9, pp. 179-186, 2014.

[98] S. M. Francis, M. E. Tan, P. R. Fung et al., "Peripheral compartment innate immune response to Haemophilus influenzae and Streptococcus pneumoniae in chronic obstructive pulmonary disease patients," Innate Immunity, vol. 19, no. 4, pp. 428-437, 2013.

[99] J. Guiot, M. Henket, J. L. Corhay, C. Moermans, and R. Louis, "Sputum biomarkers in IPF: evidence for raised gene expression and protein level of IGFBP-2, IL-8 and MMP-7," PloS One, vol. 12, no. 2, article e0171344, 2017.

[100] N. Noda, K. Matsumoto, S. Fukuyama et al., "Cigarette smoke impairs phagocytosis of apoptotic neutrophils by alveolar macrophages via inhibition of the histone deacetylase/Rac/ CD9 pathways," International Immunology, vol. 25, no. 11, pp. 643-650, 2013.

[101] T. Victoni, T. Gicquel, A. Bodin et al., "Roflumilast n-oxide associated with PGE2 prevents the neutrophil elastaseinduced production of chemokines by epithelial cells," International Immunopharmacology, vol. 30, pp. 1-8, 2016.

[102] D. Stolz, D. J. Leeming, J. H. Kristensen et al., "Systemic biomarkers of collagen and elastin turnover are associated with clinically relevant outcomes in COPD," Chest, vol. 151, no. 1, pp. 47-59, 2017.

[103] A. R. Bihlet, M. A. Karsdal, J. M. Sand et al., "Biomarkers of extracellular matrix turnover are associated with emphysema and eosinophilic-bronchitis in COPD," Respiratory Research, vol. 18, no. 1, p. 22, 2017.

[104] P. Geraghty, E. Eden, M. Pillai, M. Campos, N. G. McElvaney, and R. F. Foronjy, "alphal-antitrypsin activates protein phosphatase $2 \mathrm{~A}$ to counter lung inflammatory responses," American Journal of Respiratory and Critical Care Medicine, vol. 190, no. 11, pp. 1229-1242, 2014.

[105] I. H. Heijink, S. D. Pouwels, C. Leijendekker et al., "Cigarette smoke-induced damage-associated molecular pattern release from necrotic neutrophils triggers proinflammatory mediator release," American Journal of Respiratory Cell and Molecular Biology, vol. 52, no. 5, pp. 554-562, 2015.

[106] S. D. Pouwels, W. H. van Geffen, M. R. Jonker, H. A. Kerstjens, M. C. Nawijn, and I. H. Heijink, "Increased neutrophil expression of pattern recognition receptors during COPD exacerbations," Respirology, vol. 22, no. 2, pp. 401-404, 2017.

[107] I. Sabroe, S. K. Dower, and M. K. Whyte, "The role of Toll-like receptors in the regulation of neutrophil migration, activation, and apoptosis," Clinical Infectious Diseases, vol. 41 Supplement 7, pp. S421-S426, 2005.

[108] I. Sabroe and M. K. Whyte, “Toll-like receptor (TLR)-based networks regulate neutrophilic inflammation in respiratory disease," Biochemical Society Transactions, vol. 35, no. Part 6, pp. 1492-1495, 2007.

[109] A. Strassburg, A. Luers, and K. Dalhoff, "Decreased apoptosis of pulmonary PMN in COPD patients with communityacquired pneumonia," The Clinical Respiratory Journal, vol. 4, no. 2, pp. 111-119, 2010.

[110] E. Mortaz, I. M. Adcock, K. Ito, A. D. Kraneveld, F. P. Nijkamp, and G. Folkerts, "Cigarette smoke induces CXCL8 production by human neutrophils via activation of TLR9 receptor," The European Respiratory Journal, vol. 36, no. 5, pp. 1143-1154, 2010. 
[111] K. Guzik, J. Skret, J. Smagur et al., "Cigarette smoke-exposed neutrophils die unconventionally but are rapidly phagocytosed by macrophages," Cell Death \& Disease, vol. 2, article e131, 2011.

[112] F. Pedersen, S. Marwitz, O. Holz et al., "Neutrophil extracellular trap formation and extracellular DNA in sputum of stable COPD patients," Respiratory Medicine, vol. 109, no. 10, pp. 1360-1362, 2015.

[113] K. A. Stringer, M. Tobias, H. C. O'Neill, and C. C. Franklin, "Cigarette smoke extract-induced suppression of caspase-3-like activity impairs human neutrophil phagocytosis," American Journal of Physiology. Lung Cellular and Molecular Physiology, vol. 292, no. 6, pp. L1572-L1579, 2007.

[114] V. Brown, J. S. Elborn, J. Bradley, and M. Ennis, "Dysregulated apoptosis and NFkappaB expression in COPD subjects," Respiratory Research, vol. 10, no. 1, p. 24, 2009.

[115] D. Makris, T. Vrekoussis, M. Izoldi et al., "Increased apoptosis of neutrophils in induced sputum of COPD patients," Respiratory Medicine, vol. 103, no. 8, pp. 1130-1135, 2009.

[116] A. Obermayer, W. Stoiber, W. D. Krautgartner et al., "New aspects on the structure of neutrophil extracellular traps from chronic obstructive pulmonary disease and in vitro generation," PloS One, vol. 9, no. 5, article e97784, 2014.

[117] V. Brinkmann, U. Reichard, C. Goosmann et al., "Neutrophil extracellular traps kill bacteria," Science, vol. 303, no. 5663, pp. 1532-1535, 2004.

[118] R. Anderson, A. J. Theron, H. C. Steel, C. Durandt, G. R. Tintinger, and C. Feldman, "The beta-2-adrenoreceptor agonists, formoterol and indacaterol, but not salbutamol, effectively suppress the reactivity of human neutrophils in vitro," Mediators of Inflammation, vol. 2014, Article ID 105420, 9 pages, 2014.

[119] N. Tanabe, Y. Hoshino, S. Marumo et al., "Thioredoxin-1 protects against neutrophilic inflammation and emphysema progression in a mouse model of chronic obstructive pulmonary disease exacerbation," PloS One, vol. 8, no. 11, article e79016, 2013.

[120] C. Gao, R. Fujinawa, T. Yoshida et al., "A keratan sulfate disaccharide prevents inflammation and the progression of emphysema in murine models," American Journal of Physiology. Lung Cellular and Molecular Physiology, vol. 312, no. 2, pp. L268-L276, 2017.

[121] L. I. Kunz, N. H. Ten Hacken, T. S. Lapperre et al., "Airway inflammation in COPD after long-term withdrawal of inhaled corticosteroids," The European Respiratory Journal, vol. 49, no. 1, 2017.

[122] Y. Lei, Y. Gao, J. Chen et al., "GLCCI1 rs37973: a potential genetic predictor of therapeutic response to inhaled corticosteroids in Chinese chronic obstructive pulmonary disease patients," Scientific Reports, vol. 7, p. 42552, 2017.

[123] B. Park, G. Park, J. Kim, S. A. Lim, and K. M. Lee, "Innate immunity against Legionella pneumophila during pulmonary infections in mice," Archives of Pharmacal Research, vol. 40, no. 2, pp. 131-145, 2017.

[124] Y. Onishi, T. Kawamura, Y. Nakahara et al., "Factors associated with the relapse of cryptogenic and secondary organizing pneumonia," Respiratory Investigation, vol. 55, no. 1, pp. 10-15, 2017.

[125] S. Patamatamkul, V. Klungboonkrong, P. Praisarnti, and K. Jirakiat, "A case-control study of community-acquired Acinetobacter baumannii pneumonia and melioidosis pneumonia in northeast Thailand: an emerging fatal disease with unique clinical features," Diagnostic Microbiology and Infectious Disease, vol. 87, no. 1, pp. 79-86, 2017.

[126] R. Farah, J. Bleier, P. Gilbey, and R. Khamisy-Farah, "Common laboratory parameters for differentiating between community-acquired and healthcare-associated pneumonia," Journal of Clinical Laboratory Analysis, vol. 31, no. 1, 2017.

[127] P. R. Secor, L. A. Michaels, K. S. Smigiel et al., "Filamentous bacteriophage produced by Pseudomonas aeruginosa alters the inflammatory response and promotes noninvasive infection in vivo," Infection and Immunity, vol. 85, no. 1, 2017.

[128] V. S. Patel, R. A. Sitapara, A. Gore et al., "High mobility group box-1 mediates hyperoxia-induced impairment of Pseudomonas aeruginosa clearance and inflammatory lung injury in mice," American Journal of Respiratory Cell and Molecular Biology, vol. 48, no. 3, pp. 280-287, 2013.

[129] Y. W. Yang, Y. Z. Jiang, C. M. Hsu, and L. W. Chen, "Pseudomonas aeruginosa ventilator-associated pneumonia induces lung injury through TNF-alpha/c-Jun NH2-terminal Kinase pathways," PloS One, vol. 12, no. 1, article e0169267, 2017.

[130] Z. Piao, H. Yuan, C. Wang, and L. Shi, "IRF3 inhibits neutrophil recruitment in mice infected with Pseudomonas aeruginosa," Inflammation, 2017, [Epub ahead of print].

[131] A. Antonelli, S. Di Maggio, J. Rejman et al., "The sheddingderived soluble receptor for advanced glycation endproducts sustains inflammation during acute Pseudomonas aeruginosa lung infection," Biochimica et Biophysica Acta, vol. 1861, no. 2, pp. 354-364, 2017.

[132] M. Entezari, D. J. Weiss, R. Sitapara et al., "Inhibition of highmobility group box 1 protein (HMGB1) enhances bacterial clearance and protects against Pseudomonas aeruginosa pneumonia in cystic fibrosis," Molecular Medicine, vol. 18, pp. 477-485, 2012.

[133] Z. S. Wilson, L. B. Ahn, W. S. Serratelli et al., “Activated beta2 integrins restrict neutrophil recruitment during murine acute Pseudomonal pneumonia," American Journal of Respiratory Cell and Molecular Biology, 2017, [Epub ahead of print].

[134] R. Wilson, J. M. Cohen, R. J. Jose, C. de Vogel, H. Baxendale, and J. S. Brown, "Protection against Streptococcus pneumoniae lung infection after nasopharyngeal colonization requires both humoral and cellular immune responses," Mucosal Immunology, vol. 8, no. 3, pp. 627-639, 2015.

[135] T. J. Hommes, M. H. van Lieshout, C. van' t Veer et al., "Role of nucleotide-binding oligomerization domain-containing (NOD) 2 in host defense during pneumococcal pneumonia," PloS One, vol. 10, no. 12, article e0145138, 2015.

[136] A. Bouchon, J. Dietrich, and M. Colonna, "Cutting edge: inflammatory responses can be triggered by TREM-1, a novel receptor expressed on neutrophils and monocytes," Journal of Immunology, vol. 164, no. 10, pp. 4991-4995, 2000.

[137] M. G. Netea, T. Azam, G. Ferwerda, S. E. Girardin, S. H. Kim, and C. A. Dinarello, "Triggering receptor expressed on myeloid cells-1 (TREM-1) amplifies the signals induced by the NACHT-LRR (NLR) pattern recognition receptors," Journal of Leukocyte Biology, vol. 80, no. 6, pp. 1454-1461, 2006.

[138] T. J. Hommes, A. J. Hoogendijk, M. C. Dessing et al., "Triggering receptor expressed on myeloid cells-1 (TREM-1) improves host defence in pneumococcal pneumonia," The Journal of Pathology, vol. 233, no. 4, pp. 357-367, 2014.

[139] M. Schouten, J. D. de Boer, L. M. Kager et al., "The endothelial protein $\mathrm{C}$ receptor impairs the antibacterial response in 
murine pneumococcal pneumonia and sepsis," Thrombosis and Haemostasis, vol. 111, no. 5, pp. 970-980, 2014.

[140] H. Yang, H. J. Ko, J. Y. Yang et al., "Interleukin-1 promotes coagulation, which is necessary for protective immunity in the lung against Streptococcus pneumoniae infection," The Journal of Infectious Diseases, vol. 207, no. 1, pp. 50-60, 2013.

[141] D. C. Blok, M. H. van Lieshout, A. J. Hoogendijk et al., "Single immunoglobulin interleukin-1 receptor-related molecule impairs host defense during pneumonia and sepsis caused by Streptococcus pneumoniae," Journal of Innate Immunity, vol. 6, no. 4, pp. 542-552, 2014.

[142] A. van Belkum, N. J. Verkaik, C. P. de Vogel et al., "Reclassification of Staphylococcus aureus nasal carriage types," The Journal of Infectious Diseases, vol. 199, no. 12, pp. 18201826, 2009.

[143] S. Y. Tong, J. S. Davis, E. Eichenberger, T. L. Holland, and V. G. Fowler Jr, "Staphylococcus aureus infections: epidemiology, pathophysiology, clinical manifestations, and management," Clinical Microbiology Reviews, vol. 28, no. 3, pp. 603-661, 2015.

[144] C. K. Naber, "Staphylococcus aureus bacteremia: epidemiology, pathophysiology, and management strategies," Clinical Infectious Diseases, vol. 48 Supplement 4, pp. S231-S237, 2009.

[145] E. A. Fitzpatrick, D. You, B. Shrestha et al., "A neonatal murine model of MRSA pneumonia," PloS One, vol. 12, no. 1, article e0169273, 2017.

[146] T. Kantyka, K. Pyrc, M. Gruca et al., "Staphylococcus aureus proteases degrade lung surfactant protein A potentially impairing innate immunity of the lung," Journal of Innate Immunity, vol. 5, no. 3, pp. 251-260, 2013.

[147] K. Dietert, K. Reppe, L. Mundhenk, M. Witzenrath, and A. D. Gruber, "mCLCA3 modulates IL-17 and CXCL-1 induction and leukocyte recruitment in murine Staphylococcus aureus pneumonia," PloS One, vol. 9, no. 7, article e102606, 2014.

[148] A. Sharma, A. L. Steichen, C. N. Jondle, B. B. Mishra, and J. Sharma, "Protective role of Mincle in bacterial pneumonia by regulation of neutrophil mediated phagocytosis and extracellular trap formation," The Journal of Infectious Diseases, vol. 209, no. 11, pp. 1837-1846, 2014.

[149] Y. Miyake, E. Ishikawa, T. Ishikawa, and S. Yamasaki, "Self and nonself recognition through C-type lectin receptor, Mincle," Self Nonself, vol. 1, no. 4, pp. 310-313, 2010.

[150] C. N. Jondle, A. Sharma, T. J. Simonson, B. Larson, B. B. Mishra, and J. Sharma, "Macrophage galactose-type lectin-1 deficiency is associated with increased neutrophilia and hyperinflammation in gram-negative pneumonia," Journal of Immunology, vol. 196, no. 7, pp. 3088-3096, 2016.

[151] S. Cai, S. Batra, N. Wakamatsu, P. Pacher, and S. Jeyaseelan, "NLRC4 inflammasome-mediated production of IL-1beta modulates mucosal immunity in the lung against gramnegative bacterial infection," Journal of Immunology, vol. 188, no. 11, pp. 5623-5635, 2012.

[152] A. Achouiti, T. Vogl, C. F. Urban et al., "Myeloid-related protein-14 contributes to protective immunity in gramnegative pneumonia derived sepsis," PLoS Pathogens, vol. 8, no. 10, article e1002987, 2012.

[153] B. J. Wolff, A. J. Benitez, H. P. Desai, S. S. Morrison, M. H. Diaz, and J. M. Winchell, "Development of a multiplex
taqMan real-time PCR assay for typing of mycoplasma pneumoniae based on type-specific indels identified through whole genome sequencing," Diagnostic Microbiology and Infectious Disease, vol. 87, no. 3, pp. 203-206, 2017.

[154] H. Tanaka, "Correlation between radiological and pathological findings in patients with mycoplasma pneumoniae pneumonia," Frontiers in Microbiology, vol. 7, p. 695, 2016.

[155] L. Guo, F. Liu, M. P. Lu, Q. Zheng, and Z. M. Chen, "Increased $\mathrm{T}$ cell activation in BALF from children with mycoplasma pneumoniae pneumonia," Pediatric Pulmonology, vol. 50, no. 8, pp. 814-819, 2015.

[156] F. W. Arnold, J. Bordon, R. Fernandez-Botran et al., "Macrolide use and neutrophil function/cytokine levels in hospitalized patients with community-acquired pneumonia: a pilot study," Lung, vol. 194, no. 1, pp. 155-162, 2016.

[157] P. F. Cespedes, E. Rey-Jurado, J. A. Espinoza et al., "A single, low dose of a cGMP recombinant BCG vaccine elicits protective $\mathrm{T}$ cell immunity against the human respiratory syncytial virus infection and prevents lung pathology in mice," Vaccine, vol. 35, no. 5, pp. 757-766, 2017.

[158] Q. Xu, N. Surendran, D. Verhoeven, J. Klapa, M. Ochs, and M. E. Pichichero, "Trivalent pneumococcal protein recombinant vaccine protects against lethal Streptococcus pneumoniae pneumonia and correlates with phagocytosis by neutrophils during early pathogenesis," Vaccine, vol. 33, no. 8, pp. 993-1000, 2015.

[159] F. Pinheiro da Silva and M. C. Machado, "The dual role of cathelicidins in systemic inflammation," Immunology Letters, vol. 182, pp. 57-60, 2017.

[160] E. N. Bou Ghanem, S. Clark, X. Du et al., "The alphatocopherol form of vitamin $\mathrm{E}$ reverses age-associated susceptibility to streptococcus pneumoniae lung infection by modulating pulmonary neutrophil recruitment," Journal of Immunology, vol. 194, no. 3, pp. 1090-1099, 2015.

[161] D. Cortinovis, V. Gregorc, M. R. Migliorino et al., "New perspectives in the second-line treatment of non squamous NSCLC patients: results from a large Italian lung cancer working group," Critical Reviews in Oncology/Hematology, vol. 109, pp. 35-41, 2017.

[162] X. Zhang, W. Zhang, X. Yuan, M. Fu, H. Qian, and W. Xu, "[Corrigendum] Neutrophils in cancer development and progression: roles, mechanisms, and implications (review)," International Journal of Oncology, vol. 50, no. 2, p. 745, 2017.

[163] H. G. Vikis, A. E. Gelman, A. Franklin et al., "Neutrophils are required for 3-methylcholanthrene-initiated, butylated hydroxytoluene-promoted lung carcinogenesis," Molecular Carcinogenesis, vol. 51, no. 12, pp. 993-1002, 2012.

[164] C. Xu, C. M. Fillmore, S. Koyama et al., "Loss of Lkb1 and Pten leads to lung squamous cell carcinoma with elevated PD-L1 expression," Cancer Cell, vol. 25, no. 5, pp. 590-604, 2014.

[165] U. Meyer-Hoffert and O. Wiedow, "Neutrophil serine proteases: mediators of innate immune responses," Current Opinion in Hematology, vol. 18, no. 1, pp. 19-24, 2011.

[166] L. Chen, Q. Li, X. D. Zhou et al., "Increased proangiogenic factors, infiltrating neutrophils and CD163(+) macrophages in bronchoalveolar lavage fluid from lung cancer patients," International Immunopharmacology, vol. 20, no. 1, pp. 74-80, 2014. 
[167] T. Yamamoto, K. Kawada, Y. Itatani et al., "Loss of SMAD4 promotes lung metastasis of colorectal cancer by accumulation of CCR1+ tumor-associated neutrophils through CCL15-CCR1 axis," Clinical Cancer Research, vol. 23, no. 3, pp. 833-844, 2017.

[168] Y. Takahashi, M. Kawamura, T. Hato, M. Harada, N. Matsutani, and H. Horio, "Neutrophil-lymphocyte ratio as a prognostic marker for lung adenocarcinoma after complete resection," World Journal of Surgery, vol. 40, no. 2, pp. 365-372, 2016.

[169] L. Kasmann, L. Bolm, S. E. Schild, S. Janssen, and D. Rades, "Neutrophil-to-lymphocyte ratio predicts outcome in limited disease small-cell lung cancer," Lung, vol. 195, no. 2, pp. 217224, 2017.

[170] L. Jiang, Z. Zhao, S. Jiang et al., "Immunological markers predict the prognosis of patients with squamous non-small cell lung cancer," Immunologic Research, vol. 62, no. 3, pp. 316324, 2015.

[171] S. Cao, S. Jin, J. Shen et al., "Selected patients can benefit more from the management of etoposide and platinum-based chemotherapy and thoracic irradiation-a retrospective analysis of 707 small cell lung cancer patients," Oncotarget, vol. 8, no. 5, pp. 8657-8669, 2017.

[172] E. Jeong, S. H. Hyun, S. H. Moon, Y. S. Cho, B. T. Kim, and K. H. Lee, "Relation between tumor FDG uptake and hematologic prognostic indicators in stage I lung cancer patients following curative resection," Medicine (Baltimore), vol. 96, no. 5, article e5935, 2017.

[173] B. A. Derman, J. N. Macklis, M. S. Azeem et al., "Relationships between longitudinal neutrophil to lymphocyte ratios, body weight changes, and overall survival in patients with non-small cell lung cancer," BMC Cancer, vol. 17, no. 1, p. 141, 2017.

[174] M. Yuste, T. Fernandez-Caballero, C. Prieto et al., "Splenic $\mathrm{CD} 163+$ macrophages as targets of porcine reproductive and respiratory virus: role of Siglecs," Veterinary Microbiology, vol. 198, pp. 72-80, 2017.

[175] K. Ohnishi, M. Yamaguchi, C. Erdenebaatar et al., "Prognostic significance of CD169-positive lymph node sinus macrophages in patients with endometrial carcinoma," Cancer Science, vol. 107, no. 6, pp. 846-852, 2016.

[176] H. Laubli, O. M. Pearce, F. Schwarz et al., "Engagement of myelomonocytic Siglecs by tumor-associated ligands modulates the innate immune response to cancer," Proceedings of the National Academy of Sciences of the United States of America, vol. 111, no. 39, pp. 14211-14216, 2014.

[177] W. D. Travis, E. Brambilla, and G. J. Riely, "New pathologic classification of lung cancer: relevance for clinical practice and clinical trials," Journal of Clinical Oncology, vol. 31, no. 8, pp. 992-1001, 2013.

[178] A. S. Nagaraj, J. Lahtela, A. Hemmes et al., "Cell of origin links histotype spectrum to immune microenvironment diversity in non-small-cell lung cancer driven by mutant Kras and loss of Lkb1," Cell Reports, vol. 18, no. 3, pp. 673-684, 2017.

[179] C. F. Wu, L. Andzinski, N. Kasnitz et al., "The lack of type I interferon induces neutrophil-mediated pre-metastatic niche formation in the mouse lung," International Journal of Cancer, vol. 137, no. 4, pp. 837-847, 2015.

[180] M. Kowanetz, X. Wu, J. Lee et al., "Granulocyte-colony stimulating factor promotes lung metastasis through mobilization of Ly6G+Ly6C+ granulocytes," Proceedings of the National
Academy of Sciences of the United States of America, vol. 107, no. 50, pp. 21248-21255, 2010.

[181] R. N. Kaplan, R. D. Riba, S. Zacharoulis et al., "VEGFR1-positive haematopoietic bone marrow progenitors initiate the pre-metastatic niche," Nature, vol. 438, no. 7069, pp. 820 827,2005

[182] S. Hiratsuka, A. Watanabe, H. Aburatani, and Y. Maru, "Tumour-mediated upregulation of chemoattractants and recruitment of myeloid cells predetermines lung metastasis," Nature Cell Biology, vol. 8, no. 12, pp. 1369-1375, 2006.

[183] N. Lavender, J. Yang, S. C. Chen et al., "The Yin/Yan of CCL2: a minor role in neutrophil anti-tumor activity in vitro but a major role on the outgrowth of metastatic breast cancer lesions in the lung in vivo," BMC Cancer, vol. 17, no. 1, p. $88,2017$.

[184] S. B. Coffelt, K. Kersten, C. W. Doornebal et al., "IL-17-producing gammadelta $\mathrm{T}$ cells and neutrophils conspire to promote breast cancer metastasis," Nature, vol. 522, no. 7556, pp. 345-348, 2015.

[185] T. Bald, T. Quast, J. Landsberg et al., "Ultraviolet-radiationinduced inflammation promotes angiotropism and metastasis in melanoma," Nature, vol. 507, no. 7490, pp. 109-113, 2014.

[186] Y. M. Abadi, H. Jeon, K. C. Ohaegbulam et al., "Host b7x promotes pulmonary metastasis of breast cancer," Journal of Immunology, vol. 190, no. 7, pp. 3806-3814, 2013.

[187] C. Carbonelli, G. Rossi, and A. Cavazza, "Cryobiopsy and multidisciplinary diagnosis of idiopathic pulmonary fibrosis," Respirology, vol. 20, no. 4, p. 685, 2015.

[188] R. P. Baughman and G. Raghu, "Bronchoalveolar cellular analysis in scleroderma lung disease: does Sutton's law hold?" American Journal of Respiratory and Critical Care Medicine, vol. 177, no. 1, pp. 2-3, 2008.

[189] E. J. Lammertyn, E. Vandermeulen, H. Bellon et al., "Endstage cystic fibrosis lung disease is characterised by a diverse inflammatory pattern: an immunohistochemical analysis," Respiratory Research, vol. 18, no. 1, p. 10, 2017.

[190] K. Rydell-Tormanen, K. Andreasson, R. Hesselstrand et al., "Extracellular matrix alterations and acute inflammation; developing in parallel during early induction of pulmonary fibrosis," Laboratory Investigation, vol. 92, no. 6, pp. 917925, 2012.

[191] M. Gschwandtner, E. Strutzmann, M. M. Teixeira et al., "Glycosaminoglycans are important mediators of neutrophilic inflammation in vivo," Cytokine, vol. 91, pp. 6573, 2017.

[192] Y. Obayashi, J. Fujita, T. Nishiyama et al., "Role of carbohydrate antigens sialyl Lewis (a) (CA19-9) in bronchoalveolar lavage in patients with pulmonary fibrosis," Respiration, vol. 67, no. 2, pp. 146-152, 2000.

[193] N. Cox, D. Pilling, and R. H. Gomer, "Serum amyloid P: a systemic regulator of the innate immune response," Journal of Leukocyte Biology, vol. 96, no. 5, pp. 739-743, 2014.

[194] N. Cox, D. Pilling, and R. H. Gomer, "DC-SIGN activation mediates the differential effects of SAP and CRP on the innate immune system and inhibits fibrosis in mice," Proceedings of the National Academy of Sciences of the United States of America, vol. 112, no. 27, pp. 8385-8390, 2015.

[195] N. Cox, D. Pilling, and R. H. Gomer, "Distinct Fcgamma receptors mediate the effect of serum amyloid $\mathrm{p}$ on neutrophil adhesion and fibrocyte differentiation," Journal of Immunology, vol. 193, no. 4, pp. 1701-1708, 2014. 
[196] N. Rieber, A. Hector, M. Carevic, and D. Hartl, "Current concepts of immune dysregulation in cystic fibrosis," The International Journal of Biochemistry \& Cell Biology, vol. 52, pp. 108-112, 2014.

[197] D. L. Forrester, H. L. Barr, A. Fogarty, and A. Knox, "sTREM1 is elevated in cystic fibrosis and correlates with proteases," Pediatric Pulmonology, vol. 52, no. 4, pp. 467-471, 2017.

[198] J. Eckrich, U. M. Zissler, F. Serve et al., "Airway inflammation in mild cystic fibrosis," Journal of Cystic Fibrosis, vol. 16, no. 1, pp. 107-115, 2017.

[199] S. A. Ingersoll, J. Laval, O. A. Forrest et al., "Mature cystic fibrosis airway neutrophils suppress T cell function: evidence for a role of arginase 1 but not programmed death-ligand 1," Journal of Immunology, vol. 194, no. 11, pp. 5520-5528, 2015.

[200] S. Zheng, A. B. Kummarapurugu, D. K. Afosah et al., "2-O, 3$\mathrm{O}$ desulfated heparin blocks high mobility group box 1 release by inhibition of p300 acetyltransferase activity," American Journal of Respiratory Cell and Molecular Biology, vol. 56, no. 1, pp. 90-98, 2017.

[201] B. A. Flitter, K. L. Hvorecny, E. Ono et al., "Pseudomonas aeruginosa sabotages the generation of host proresolving lipid mediators," Proceedings of the National Academy of Sciences of the United States of America, vol. 114, no. 1, pp. 136-141, 2017.

[202] H. P. Ng, Y. Zhou, K. Song, C. A. Hodges, M. L. Drumm, and G. Wang, "Neutrophil-mediated phagocytic host defense defect in myeloid Cftr-inactivated mice," PloS One, vol. 9, no. 9, article e106813, 2014.

[203] P. Duchesneau, R. Besla, M. F. Derouet et al., "Partial restoration of CFTR function in cftr-null mice following targeted cell replacement therapy," Molecular Therapy, vol. 25, no. 3, pp. 654-665, 2017.

[204] A. Hector, M. Kormann, J. Kammermeier et al., "Expression and regulation of interferon-related development regulator1 in cystic fibrosis neutrophils," American Journal of Respiratory Cell and Molecular Biology, vol. 48, no. 1, pp. 71-77, 2013.

[205] G. Y. Chen, C. Chen, L. Wang, X. Chang, P. Zheng, and Y. Liu, "Cutting edge: broad expression of the FoxP3 locus in epithelial cells: a caution against early interpretation of fatal inflammatory diseases following in vivo depletion of FoxP3expressing cells," Journal of Immunology, vol. 180, no. 8, pp. 5163-5166, 2008.

[206] D. Shin, G. Lee, S. Lee et al., “Adenovirus-mediated Foxp3 expression in lung epithelial cells ameliorates acute radiation-induced pneumonitis in mice," Gene Therapy, vol. 24, no. 2, pp. 104-112, 2017.

[207] G. C. Mouradian, R. Gaurav, S. Pugliese et al., "Superoxide dismutase 3 R213G single-nucleotide polymorphism blocks murine bleomycin-induced fibrosis and promotes resolution of inflammation," American Journal of Respiratory Cell and Molecular Biology, vol. 56, no. 3, pp. 362-371, 2017.

[208] F. Yang, Y. Cao, J. Zhang, T. You, and L. Zhu, "Glaucocalyxin A improves survival in bleomycin-induced pulmonary fibrosis in mice," Biochemical and Biophysical Research Communications, vol. 482, no. 1, pp. 147-153, 2017.

[209] J. S. Elborn, A. Horsley, G. MacGregor et al., "Phase I studies of acebilustat: biomarker response and safety in patients with cystic fibrosis," Clinical and Translational Science, vol. 10, no. 1, pp. 28-34, 2017.
[210] E. Hayes, K. Pohl, N. G. McElvaney, and E. P. Reeves, "The cystic fibrosis neutrophil: a specialized yet potentially defective cell," Archivum Immunologiae et Therapiae Experimentalis (Warsz), vol. 59, no. 2, pp. 97-112, 2011.

[211] E. P. Reeves, N. Banville, D. M. Ryan et al., "Intracellular secretory leukoprotease inhibitor modulates inositol 1,4,5triphosphate generation and exerts an anti-inflammatory effect on neutrophils of individuals with cystic fibrosis and chronic obstructive pulmonary disease," BioMed Research International, vol. 2013, p. 560141, 2013.

[212] O. J. McElvaney, N. O'Reilly, M. White et al., "The effect of the decoy molecule PA401 on CXCL8 levels in bronchoalveolar lavage fluid of patients with cystic fibrosis," Molecular Immunology, vol. 63, no. 2, pp. 550-558, 2015.

[213] T. G. Boyce, "More on viral bronchiolitis in children," The New England Journal of Medicine, vol. 375, no. 12, pp. 1199-1200, 2016.

[214] J. L. Simpson, S. Phipps, and P. G. Gibson, "Inflammatory mechanisms and treatment of obstructive airway diseases with neutrophilic bronchitis," Pharmacology \& Therapeutics, vol. 124, no. 1, pp. 86-95, 2009.

[215] E. C. Cavallaro, K. K. Liang, M. D. Lawrence, K. D. Forsyth, and D. L. Dixon, "Neutrophil infiltration and activation in bronchiolitic airways are independent of viral etiology," Pediatric Pulmonology, vol. 52, no. 2, pp. 238-246, 2017.

[216] D. L. Dixon, K. M. Griggs, K. D. Forsyth, and A. D. Bersten, "Lower interleukin-8 levels in airway aspirates from breastfed infants with acute bronchiolitis," Pediatric Allergy and Immunology, vol. 21, no. 4 Pt 2, pp. e691e696, 2010.

[217] E. Pace, M. Ferraro, A. Giarratano, C. Cipollina, and M. Gjomarkaj, "TLR4 up-regulation and reduced Foxp3 expression in mechanically ventilated smokers with obstructive chronic bronchitis," Copd, vol. 10, no. 2, pp. 147-155, 2013.

[218] K. Sahlander, K. Larsson, and L. Palmberg, "Altered innate immune response in farmers and smokers," Innate Immunity, vol. 16, no. 1, pp. 27-38, 2010.

[219] K. Sahlander, K. Larsson, and L. Palmberg, "Daily exposure to dust alters innate immunity," PloS One, vol. 7, no. 2, article e31646, 2012.

[220] W. Jiang, T. Wang, L. Li, W. Ji, Y. Wang, and Y. Yan, "Impact of bacteria in nasal aspirates on disease severity of bronchiolitis," Infectious Diseases, vol. 48, no. 1, pp. 82-86, 2016.

[221] A. B. Chang, J. W. Upham, I. B. Masters et al., "Protracted bacterial bronchitis: the last decade and the road ahead," Pediatric Pulmonology, vol. 51, no. 3, pp. 225-242, 2016.

[222] T. Matsumoto, M. Fujita, R. Hirano et al., "Chronic Pseudomonas aeruginosa infection-induced chronic bronchitis and emphysematous changes in CCSP-deficient mice," International Journal of Chronic Obstructive Pulmonary Disease, vol. 11, pp. 2321-2327, 2016.

[223] M. C. Suarez-Arrabal, C. Mella, S. M. Lopez et al., "Nasopharyngeal bacterial burden and antibiotics: influence on inflammatory markers and disease severity in infants with respiratory syncytial virus bronchiolitis," The Journal of Infection, vol. 71, no. 4, pp. 458-469, 2015.

[224] L. A. Borthwick, M. I. Suwara, S. C. Carnell et al., "Pseudomonas aeruginosa induced airway epithelial injury drives fibroblast activation: a mechanism in chronic lung allograft dysfunction," American Journal of Transplantation, vol. 16, no. 6, pp. 1751-1765, 2016. 
[225] M. A. Farrag and F. N. Almajhdi, "Human respiratory syncytial virus: role of innate immunity in clearance and disease progression," Viral Immunology, vol. 29, no. 1, pp. 11-26, 2016.

[226] B. Cortjens, O. J. de Boer, R. de Jong et al., "Neutrophil extracellular traps cause airway obstruction during respiratory syncytial virus disease," The Journal of Pathology, vol. 238, no. 3, pp. 401-411, 2016.

[227] C. Schnoeller, X. Roux, D. Sawant et al., "Attenuated Bordetella pertussis vaccine protects against respiratory syncytial virus disease via an IL-17-dependent mechanism," American Journal of Respiratory and Critical Care Medicine, vol. 189, no. 2, pp. 194-202, 2014.

[228] P. M. Fisichella, C. S. Davis, E. Lowery, L. Ramirez, R. L. Gamelli, and E. J. Kovacs, “Aspiration, localized pulmonary inflammation, and predictors of early-onset bronchiolitis obliterans syndrome after lung transplantation," Journal of the American College of Surgeons, vol. 217, no. 1, pp. 90100, 2013, discussion 100-1.

[229] J. Eckrich, E. Herrmann, S. Voss, R. Schubert, S. Zielen, and M. Rosewich, "Short-term variation of lung function and airway inflammation in children and adolescents with bronchiolitis obliterans," Lung, vol. 194, no. 4, pp. 571-579, 2016.

[230] E. Vandermeulen, E. Lammertyn, S. E. Verleden et al., "Immunological diversity in phenotypes of chronic lung allograft dysfunction: a comprehensive immunohistochemical analysis," Transplant International, vol. 30, no. 2, pp. 134143, 2017.

[231] V. Tiriveedhi, M. Takenaka, N. J. Sarma, A. G. Gelman, and T. Mohanakumar, "Anti-major histocompatibility complexinduced obliterative airway disease: selective role for CD4 and CD8 T cells in inducing immune responses to self-antigens," The Journal of Heart and Lung Transplantation, vol. 32, no. 7, pp. 714-722, 2013.

[232] V. Tiriveedhi, M. Takenaka, S. Ramachandran et al., "T regulatory cells play a significant role in modulating MHC class I antibody-induced obliterative airway disease," American Journal of Transplantation, vol. 12, no. 10, pp. 2663-2674, 2012.

[233] R. Krebs, J. M. Tikkanen, J. O. Ropponen et al., "Critical role of VEGF-C/VEGFR-3 signaling in innate and adaptive immune responses in experimental obliterative bronchiolitis," The American Journal of Pathology, vol. 181, no. 5, pp. 1607-1620, 2012.

[234] J. L. Todd, X. Wang, S. Sugimoto et al., "Hyaluronan contributes to bronchiolitis obliterans syndrome and stimulates lung allograft rejection through activation of innate immunity," American Journal of Respiratory and Critical Care Medicine, vol. 189, no. 5, pp. 556-566, 2014.

[235] Y. Zhao, D. J. LaPar, J. Steidle et al., “Adenosine signaling via the adenosine $2 \mathrm{~B}$ receptor is involved in bronchiolitis obliterans development," The Journal of Heart and Lung Transplantation, vol. 29, no. 12, pp. 1405-1414, 2010.

[236] C. L. Lau, Y. Zhao, I. L. Kron et al., "The role of adenosine A2A receptor signaling in bronchiolitis obliterans," The Annals of Thoracic Surgery, vol. 88, no. 4, pp. 1071-1078, 2009. 


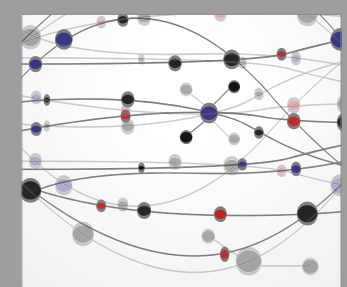

The Scientific World Journal
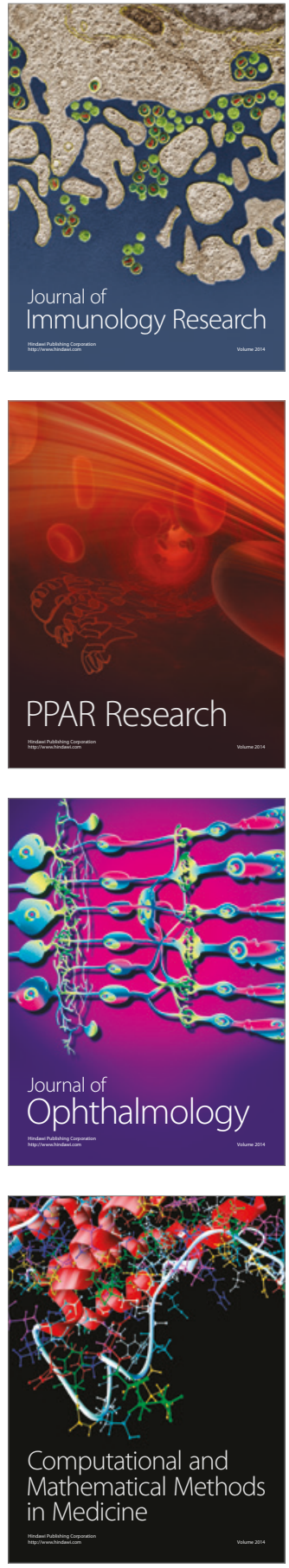

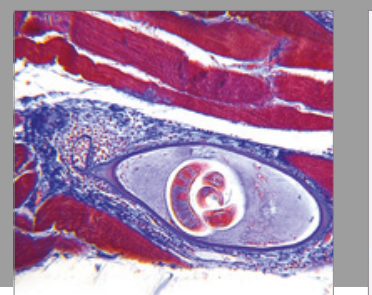

Gastroenterology Research and Practice
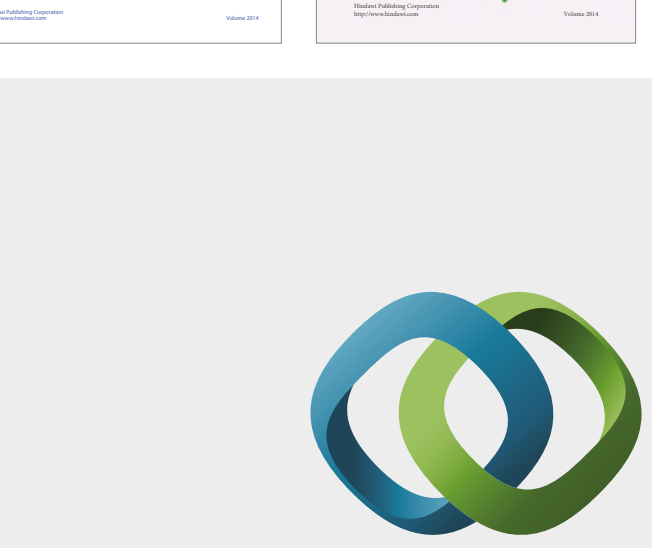

\section{Hindawi}

Submit your manuscripts at

https://www.hindawi.com
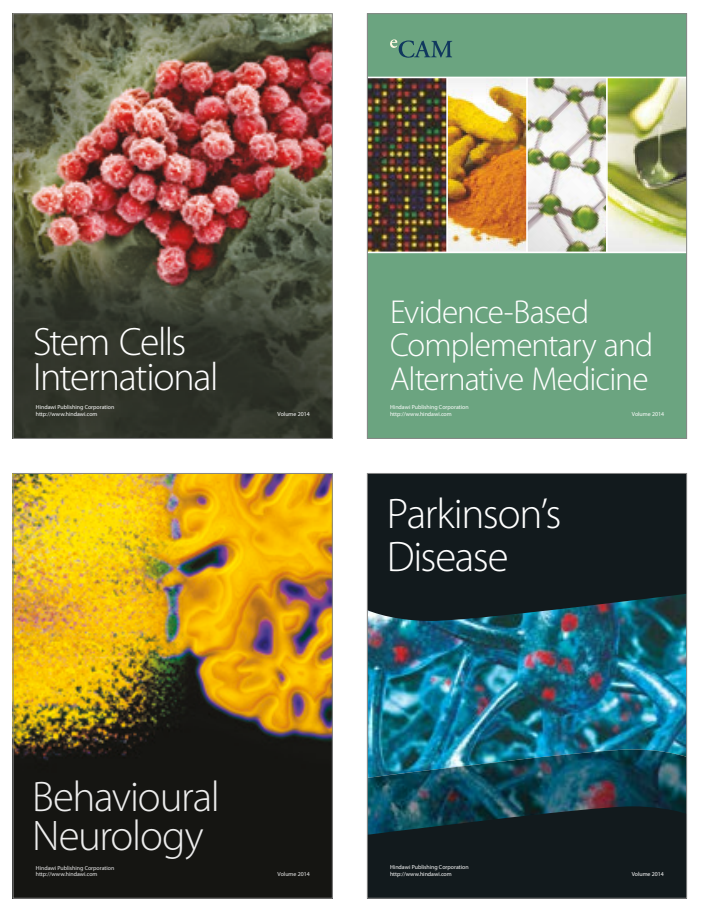
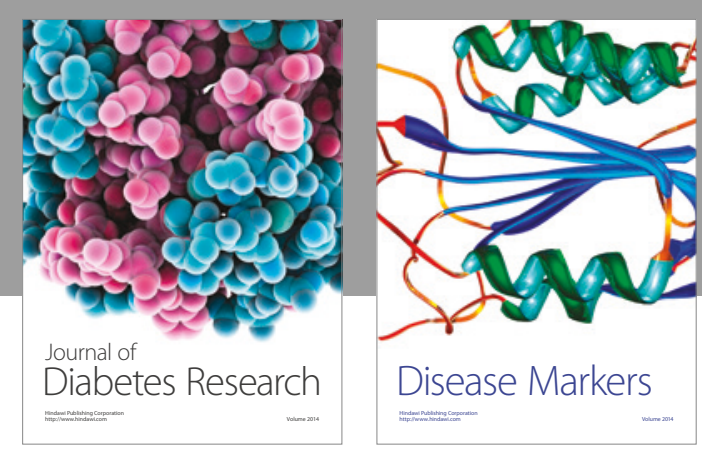

Disease Markers
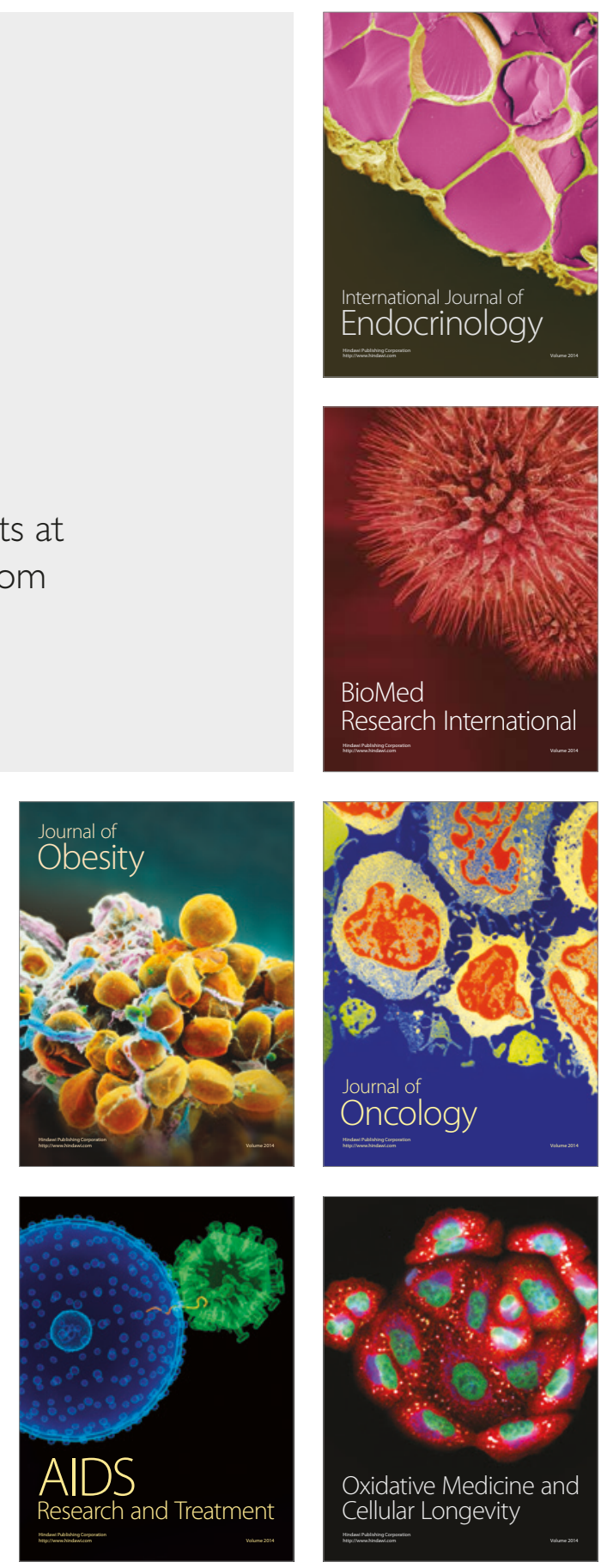